Preprint typeset in JHEP style - HYPER VERSION

\title{
Conway Subgroup Symmetric Compactifications Of Heterotic String
}

\author{
Jeffrey A. Harvey ${ }^{1}$ and Gregory W. Moore ${ }^{2}$ \\ ${ }^{1}$ Enrico Fermi Institute and Department of Physics \\ University of Chicago \\ 5620 Ellis Ave., Chicago IL 60637 \\ 2 NHETC and Department of Physics and Astronomy, Rutgers University \\ 126 Frelinghuysen Rd., Piscataway NJ 08855, USA \\ j-harvey@uchicago.edu, gmoore@physics.rutgers.edu
}

\begin{abstract}
We investigate special compactifications of the heterotic string for which the space of half-BPS states is, in a natural way, a representation of various subgroups of the Conway group. These compactifications provide a useful framework for analyzing the action of some of the large symmetry groups appearing in discussions of Moonshine in the physics literature. We investigate toroidal compactifications of heterotic string with sixteen supersymmetries as well as asymmetric toroidal orbifolds with $N=2$ supersymmetry in four dimensions that arise as $K 3 \times T^{2}$ compactifications. The latter Conway subgroup symmetric compactifications of the heterotic string might have some interesting implications for D-brane bound states on Calabi-Yau manifolds.
\end{abstract}

December 22, 2017

Keywords: Superstring and Heterotic Strings, Discrete Symmetries, Supersymmetry and Duality. 


\section{Contents}

1. Introduction And Conclusion 2

2. Conway Subgroup Symmetric Compactifications

2.1 Recollections On Narain Compactification

2.2 A Lattice Lemma

3. Examples Of CSS Compactifications 8

3.1 Right-moving Lattice $\mathfrak{F}_{R}$ And Orbifold Action On Right-Movers

3.2 Left-moving Lattice $\mathfrak{F}_{L}$

3.3 Orbifold Action On Left-Movers 10

3.4 Massless States In The Untwisted Sector

3.5 Massless States In The Twisted Sectors 12

3.6 The Fate Of Massless Charged Hypermultiplets 14

3.7 Computation Of Discriminant Groups, $\mathcal{N}_{1,0}$ And $\mathcal{L}_{1,0}$

3.8 The Discrete Symmetries 17

3.9 Summary Of The Six Models 17

4. Implications Of Heterotic-Type II Duality 18

4.1 Finding Type II Duals 18

4.2 Auto-Equivalences Of The Derived Category 19

4.3 Categories Of D-Branes 20

A. Computation Of The Untwisted Sector For The Four-Dimensional CSS Models 21

B. The Binary Golay Code, The Steiner System $S(5,8,24)$, And The Leech Lattice 22

G. Details On Lattice Computations: HM222 25

C.1 Construction Of $\Gamma^{22,6}$

D. Details On Lattice Computations: HM224 27

D.1 Construction Of $\Gamma^{22,6}$

D.2 Doomed To Fail? 29

D.3 The Invariant Lattice $\Gamma^{g}$ And Its Discriminant Group 29

D.4 The Massless Charged Twisted Sector Ground States 30

E. A Case Of Mistaken Moonshine 31

E.1 What Is Moonshine? 32

E.2 Virtual Representations 33

E.3 $d=4$ : Mistaken Moonshine 34 


\section{Introduction And Conclusion}

This paper discusses discrete symmetries of some special toroidal, and toroidal orbifold, compactifications of the heterotic string. The construction of the toroidal compactifications is described in section 2.2. The construction is a simple application of results of Nikulin [62]. There are three motivations for discussing this topic.

The first motivation concerns Moonshine, old and new. As is very well known, the original Moonshine conjectures of Conway and Norton [17], associated with the Monster group and the modular $j$ function, led to some very interesting developments in string theory and in mathematics including the construction of an explicit Vertex Operator Algebra (VOA) or holomorphic Conformal Field Theory (CFT) with Monster symmetry in [30] and the proof of the genus zero property of Monstrous Moonshine by Borcherds [7]. In recent years various new types of Moonshine conjectures have again caught the attention of string theorists and mathematicians [12, 13, 26, 27, 40]. Some of these conjectures have now been proved [25, 33, 37], but the proofs are not constructive and the full implications of these new Moonshine results both for string theory and for mathematics remain to be understood. In particular, it remains to be seen whether or not there are VOA and/or CFT constructions which underlie these new examples of Moonshine. The present paper studies CFTs associated with heterotic string theory compactifications with large discrete symmetries and is thus potentially relevant to the new Moonshine phenomena.

A second motivation for this paper is the evidence for Moonshine associated to the Mathieu group $M_{24}$ that was recently observed in the computation of refined DT-type invariants for K3 surfaces [48. The relevance of the present paper to the considerations of [48] is provided courtesy of heterotic-type II duality. (For a review, see [3].) Viewed through the lens of heterotic-type II duality, the invariants of [48] are counting perturbative BPS states in the heterotic dual so it is natural to ask whether there are heterotic compactifications in which the perturbative BPS states form a representation for $M_{24}$ or closely related groups. In Appendix $\mathrm{E}$ of this paper we will give some strong evidence that, regrettably, there is in fact no natural $M_{24}$ representation underlying the DT invariants computed in [48]. The $O(4)$ representations studied in 48] are organized by $p^{2}$, rather than $p$ (where $p$ is the Narain lattice vector). For this reason they are not sensitive to the specific crystal structure of the Narain lattice and the degeneracies are more simply understood in terms of the natural $O(20) \times O(4)$ symmetry of dimensional reduction to six noncompact dimensions: The $O(4)$ is the massless little group in six dimensions while the $O(20)$ rotates all the "internal" left-moving bosonic fields of the heterotic string.

In spite of this negative, and disappointing, conclusion our considerations do raise the interesting issue of the relation of symmetries present in a worldsheet, or perturbative, 
analysis of a string theory to symmetries of nonperturbative states. For example, it was shown in 31] that any automorphism of a (smooth) K3 sigma model is a subgroup of the Conway group stabilizing a four-plane in the Leech lattice. It is interesting to ask whether this classification, based on the worldsheet conformal structure, also extends to symmetries of the full string theory including nonperturbative states. The study of heterotic string on $T^{4}$ provides a tool to start addressing this question since perturbative BPS states in the heterotic string (first studied in [19]) map to nonperturbative states of the type II string on $K 3$ under string duality. Indeed, heterotic/type II duality implies that in this case the worldsheet symmetries identified in [31] do indeed extend to the nonperturbative BPS sector. In fact, the relevant mathematical version of this statement has been rigorously proven in [44].

The third and final motivation for this paper is based on a very natural approach to giving a conceptual framework for the Mathieu Moonshine of [27]. The basic idea is to identify $M_{24}$ as an automorphism group (or a distinguished subgroup thereof) of the algebra of BPS states discussed in [38, 39]. In the present context there are actually several ways in which this general idea could be implemented. One might try to consider the algebra of BPS states of the type IIA string compactified to six Minkowski dimensions along a K3 surface. This would involve considerations of wrapped D4-D2-D0 brane bound states (or objects in the derived category of K3) mentioned above. Again using the lens of heterotic-type II duality, one might try to consider instead the algebra of perturbative BPS states of the toroidally compactified heterotic dual theory. The latter has the advantage that the algebra then has a concrete and computable definition in terms of vertex operator algebras [39]. (The "correspondence conjecture" of [39] asserts that the algebra constructed using vertex operators has a geometrical construction on the type II side, the latter being inspired by work of Nakajima.) This paper focuses on the heterotic string. Unsurprisingly, in view of the work of [31], we will show that it is possible to formulate heterotic toroidal CFTs with discrete symmetries related to subgroups of the Conway group. As stressed by [31] the relevant list of symmetries is not naturally related to $M_{24}$. That leaves the logical possibility that there are extra symmetries of the BPS sector, not present in the full CFT. Such "extra symmetries" might well exist and we have not ruled them out. Their existence would be extremely interesting. However, we can say that the the considerations of Appendix $\mathrm{E}$ indicate that $M_{24}$ cannot be such a group of "extra symmetries," even when restricting attention to the BPS sector.

From the viewpoint of explaining Mathieu Moonshine our results are thus largely negative. We hasten to add that our considerations do not exclude the possible relevance of BPS algebras to the explanation of Mathieu Moonshine. Rather, they narrow the search for situations in which such algebras could be relevant. For example, one natural and unexplored direction is to consider perturbative type IIA BPS states in a compactification on $\mathbb{R}^{1,4} \times K 3 \times S^{1}$ or (what seems to us a more likely scenario) on $\mathbb{R}^{1,1} / I I^{1,1} \times K 3 \times \mathfrak{X}$ for a suitable compact space $\mathfrak{X}$. It seems to us that this is a potentially interesting line of future research.

Given the toroidal compactifications with large discrete symmetries related to the Conway group we can still do some interesting things with them, again in light of heterotic/type 
II duality. Namely, we can consider orbifolds of the heterotic string to $T^{2} \times K 3$ compactifications that still preserve large subgroups of the Conway group. We then expect these to imply interesting generalizations of the automorphisms of the derived category of $\mathrm{K} 3$ explored in 44. Motivated by this we have constructed several such (asymmetric) orbifolds. As it turns out, this is no easy task. The methods and results are outlined in section 3 and some of the many details are given in Appendices A,B,C,D. The key properties of the models are summarized in Tables 11 and 2 below. We suggest some potential implications of our results for Calabi-Yau geometry and directions for further research in Section $⿴$.

\section{Acknowledgements}

We thank T. Banks, T. Bridgeland, M. Cheng, E. Diaconescu, R. Donagi, G. Höhn, G. Mason, D. Morrison, K. Narain, N. Paquette, D. Park, B. Pioline, M. Rocek, N. Seiberg, and W. Taylor for very useful discussions and correspondence. JH and GM gratefully acknowledge the hospitality of the Aspen Center for Physics (under NSF Grant No. PHY-1066293) where this work was initiated. They also thank the Perimeter Institute for Theoretical Physics and the Institute For Advanced Study for hospitality. We have made use of the GAP package for finite group theory in our analysis [34] as well as the Magma Computer Algebra system which was made available through a grant from the Simons Foundation. JH acknowledges support from the NSF ${ }^{1}$ under grant PHY 1520748 and from the Simons Foundation (\#399639). GM is supported by the DOE under grant DOE-SC0010008 to Rutgers.

\section{Conway Subgroup Symmetric Compactifications}

\subsection{Recollections On Narain Compactification}

We consider toroidal compactification of the heterotic string on

$$
\mathbb{M}^{1,1+d} \times T^{8-d}
$$

As is well-known, the lattice of zero modes of the worldsheet coordinates forms an even unimodular lattice of signature $(24-d ; 8-d)$ embedded in a fixed pseudo-Euclidean space of the same signature. We denote the lattice as

$$
\Gamma^{24-d ; 8-d} \subset \mathbb{R}^{24-d ; 8-d},
$$

or sometimes just by $\Gamma$ when $d$ is understood. The projections of a vector $p \in \Gamma^{24-d ; 8-d}$ onto the definite subspaces $\mathbb{R}^{24-d ; 0}$ and $\mathbb{R}^{0 ; 8-d}$, will be denoted by $p_{L}$ and $p_{R}$, respectively. ${ }^{2}$

\footnotetext{
${ }^{1}$ Any opinions, findings, and conclusions or recommendations expressed in this material are those of the author(s) and do not necessarily reflect the views of the National Science Foundation.

${ }^{2}$ We will generically denote vectors in $\mathbb{R}^{24-d ; 8-d}$ by $(x ; y)$ where the projection to the negative definite subspace $\mathbb{R}^{24-d ; 0}$ is $x$ and the projection to the positive definite subspace $\mathbb{R}^{0 ; 8-d}$ is $y$. If $L$ is a positive definite lattice embedded in Euclidean space $\mathbb{R}^{24-d}$ we denote by $(L ; 0)$ the sublattice of $\mathbb{R}^{24-d ; 8-d}$ consisting of vectors $(\ell ; 0)$ with $\ell \in L$. Thus, as an abstract lattice $(L ; 0)$ is isomorphic to $L(-1)$. We let $I I^{p, q}$ denote the even unimodular lattice of signature $(p, q)$, so $p-q=0 \bmod 8$ and we take $p, q>0$.
} 
Orthogonal rotations of these definite subspaces are symmetries of the worldsheet conformal field theory and hence the moduli space of toroidal theories is the Narain space

$$
O_{\mathbb{Z}}\left(I I^{24-d ; 8-d}\right) \backslash O_{\mathbb{R}}(24-d ; 8-d) /\left(O_{\mathbb{R}}(24-d) \times O_{\mathbb{R}}(8-d)\right) .
$$

At points in Narain moduli space where one of the groups

$$
G_{L}=\operatorname{Aut}\left(\Gamma^{24-d ; 8-d}\right) \cap O_{\mathbb{R}}(24-d) \quad G_{R}=\operatorname{Aut}\left(\Gamma^{24-d ; 8-d}\right) \cap O_{\mathbb{R}}(8-d)
$$

is nontrivial there are orbifold singularities, and there is a lift of crystal symmetry $G_{L} \times G_{R}$ to groups $\tilde{G_{L}} \times \tilde{G_{R}}$ which are global symmetries of the worldsheet theory 41. A famous example of such discrete symmetries are the Weyl-group symmetries at subloci of Narain moduli space at which there are enhanced non-abelian gauge symmetry. We are interested here in other kinds of crystal symmetries that are not of this type. We call them "Conway subgroup symmetries."

The simplest example of a Conway subgroup symmetry arises for $d=0$, that is, toroidal compactification of the heterotic string to two spacetime dimensions. A distinguished point in Narain moduli space corresponds to the embedded even unimodular lattice: ${ }^{3}$

$$
\Gamma_{*}^{24 ; 8}:=(\Lambda ; 0) \oplus\left(0 ; \Gamma_{8}\right) .
$$

The crystal symmetry for this compactification is $\mathrm{Co}_{0} \times W\left(E_{8}\right)$, where $\mathrm{Co}_{0}$ is the Conway group, by definition the group of automorphisms of the Leech lattice $\Lambda$ [16].

Most of what follows in this paper applies equally well to the other 23 distinguished points obtained by replacing the Leech lattice by one of the Niemeier lattices. Indeed this democracy among the 24 Niemeier lattices played an important role in [14, 46].

\subsection{A Lattice Lemma}

Our first goal is to construct lattices $\Gamma^{24-d ; 8-d}$ which do not have enhanced gauge symmetry, that is have no points $\left(p_{L}, 0\right) \in \Gamma^{24-d ; 8-d}$ with $p_{L}^{2}=2$ and yet have enhanced discrete symmetries. To do so we need need the following result in the theory of lattices:

Lemma: Suppose we have two primitively embedded sub-lattices $\mathfrak{F}_{R} \subset \Gamma_{8}$ and $\mathfrak{F}_{L} \subset \Lambda$ where $\Gamma_{8}$ is the $E_{8}$ lattice and $\Lambda$ is the Leech lattice, and suppose that $\mathfrak{F}_{R}$ and $\mathfrak{F}_{L}$ are of rank $d$ and isometric. Then we can construct an even unimodular lattice $\Gamma \cong I I^{24-d ; 8-d}$ and an embedding of $\Gamma$ into $\mathbb{R}^{24-d ; 8-d}$ such that

$$
\operatorname{Fix}\left(\mathfrak{F}_{L}\right) \times \operatorname{Fix}\left(\mathfrak{F}_{R}\right) \subset \mathrm{Co}_{0} \times W\left(E_{8}\right)
$$

is a crystallographic symmetry of $\Gamma$ with $\operatorname{Fix}\left(\mathfrak{F}_{L}\right) \subset O(24-d)$ and $\operatorname{Fix}\left(\mathfrak{F}_{R}\right) \subset O(8-d)$.

Proof: Let us begin by recalling a standard fact from lattice theory. (See [56, 62] for further explanation.) If $\mathfrak{L}$ is an even integral lattice then we can define the discriminant group

\footnotetext{
${ }^{3}$ In what follows we will use a concrete model of the $E_{8}$ lattice $\Gamma_{8} \subset \mathbb{R}^{8}$ as the set of vectors $\left(y_{1}, \ldots, y_{8}\right)$ where the coordinates are either all integral or all integral plus $1 / 2$ and in both cases the sum of the coordinates is even. We will also use a similar description of the Leech lattice $\Lambda$. See Appendix B for some relevant definitions.
} 
$\mathcal{D}_{ \pm}(\mathfrak{L})$ as follows. We consider the dual lattice $\mathfrak{L}^{\vee} \subset \mathfrak{L} \otimes \mathbb{Q}$ and the finite abelian group $\mathcal{D}(\mathfrak{L}):=\mathfrak{L}^{\vee} / \mathfrak{L}$. This finite group inherits a pair of quadratic functions $q_{ \pm}: \mathcal{D}(\mathfrak{L}) \rightarrow \mathbb{Q} / 2 \mathbb{Z}$ using the inner product on $\mathfrak{L}^{\vee}$ :

$$
q_{ \pm}(\bar{v}):= \pm v^{2} \bmod 2 \mathbb{Z}
$$

where $\bar{v}$ has a representative $v \in \mathfrak{L}^{\vee}$. That is $\bar{v}=[v]$. Note that $q_{ \pm}(\bar{v})$ does not depend on the representative $v$. The phrase "quadratic function" means that

$$
q_{ \pm}\left(\bar{v}_{1}+\bar{v}_{2}\right)-q_{ \pm}\left(\bar{v}_{1}\right)-q_{ \pm}\left(\bar{v}_{2}\right)+q_{ \pm}(0):=2 b_{ \pm}\left(\bar{v}_{1}, \bar{v}_{2}\right)
$$

defines a bilinear form $b_{ \pm}$valued in $\mathbb{Q} / \mathbb{Z}$. We denote by $\mathcal{D}_{ \pm}(\mathfrak{L})$ the finite group equipped with the quadratic function $q_{ \pm}$. Sometimes we will write $q_{\mathcal{D}_{ \pm}(\mathfrak{L})}$ when we want to emphasize the lattice.

Now suppose that $\Gamma$ is an even unimodular lattice and $\mathfrak{F} \subset \Gamma$ is a primitively embedded sublattice. Primitively embedded means that the Abelian group $\Gamma / \mathfrak{F}$ is free, that is it has no elements of finite order. Then in [56, 62, it is shown that there is a canonical isometric isomorphism

$$
\psi: \mathcal{D}_{+}(\mathfrak{F}) \rightarrow \mathcal{D}_{-}\left(\mathfrak{F}^{\perp}\right)
$$

where "isometric" means $q_{-}(\psi(\bar{v}))=q_{+}(\bar{v})$.

In particular, given a primitively embedded sublattice $\mathfrak{F}_{L} \subset \Lambda$ there is an isomorphism of discriminant groups

$$
\psi_{L}: \mathcal{D}_{+}\left(\mathfrak{F}_{L}^{\perp}\right) \rightarrow \mathcal{D}_{-}\left(\mathfrak{F}_{L}\right)
$$

where $\mathfrak{F}_{L}^{\perp} \subset \Lambda$ is the orthogonal complement of $\mathfrak{F}_{L}$ within $\Lambda$. Similarly, there is an isomorphism

$$
\psi_{R}: \mathcal{D}_{-}\left(\mathfrak{F}_{R}\right) \rightarrow \mathcal{D}_{+}\left(\mathfrak{F}_{R}^{\perp}\right) .
$$

Now we define a quadratic space $V$ by

$$
V=\Lambda \otimes \mathbb{Q} \oplus \Gamma_{8} \otimes \mathbb{Q} \cong \mathbb{Q}^{24 ; 8}
$$

writing vectors as $(x ; y)$ with $x \in \Lambda \otimes \mathbb{Q}$ and $y \in \Gamma_{8} \otimes \mathbb{Q}$ with

$$
(x ; y)^{2}=-x^{2}+y^{2}
$$

We can of course further extend scalars from $\mathbb{Q}$ to $\mathbb{R}$.

Similarly define the subspaces of $V$ :

$$
W_{d}:=\mathfrak{F}_{L}^{\perp} \otimes \mathbb{Q} \oplus \mathfrak{F}_{R}^{\perp} \otimes \mathbb{Q} \subset V
$$

$W_{d}$ is a quadratic space isomorphic to $\mathbb{Q}^{24-d ; 8-d} .{ }^{4}$

Now we choose an isometry between $\mathfrak{F}_{L}$ and $\mathfrak{F}_{R}$. This will induce an isomorphism

$$
\psi_{R L}: \mathcal{D}_{-}\left(\mathfrak{F}_{L}\right) \rightarrow \mathcal{D}_{-}\left(\mathfrak{F}_{R}\right)
$$

\footnotetext{
${ }^{4}$ Note that which subspace of $V$ we get depends on $\mathfrak{F}_{L}$ and $\mathfrak{F}_{R}$, and hence is less canonical than $V$ itself.
} 
allowing us to define an isomorphism

$$
\psi: \mathcal{D}_{+}\left(\mathfrak{F}_{L}^{\perp}\right) \rightarrow \mathcal{D}_{+}\left(\mathfrak{F}_{R}^{\perp}\right)
$$

by

$$
\psi=\psi_{R} \circ \psi_{R L} \circ \psi_{L} .
$$

Next define an embedded lattice in $W_{d}$ :

$$
\tilde{\Gamma}:=\left(\mathfrak{F}_{L}^{\perp}\right)^{\vee} \oplus\left(\mathfrak{F}_{R}^{\perp}\right)^{\vee} \subset W_{d}
$$

and also define a sublattice of $\tilde{\Gamma}$ :

$$
\Gamma \subset \tilde{\Gamma}
$$

to be the set of vectors $(x ; y) \in \tilde{\Gamma}$ such that $\psi(\bar{x})=\bar{y}$.

It is a standard result that the lattice $\Gamma$ constructed this way is even unimodular [56, 62], but for completeness let us recall the proof. The fact that it is an integral even lattice is easy to demonstrate: If $(x ; y) \in \Gamma$ then

$$
\begin{aligned}
(x ; y)^{2} \bmod 2 & =-x^{2}+y^{2} \bmod 2 \\
& =-q_{\mathcal{D}_{+}\left(\mathfrak{F}_{L}^{\perp}\right)}(\bar{x})+q_{\mathcal{D}_{+}\left(\mathfrak{F}_{R}^{\perp}\right)}(\bar{y}) \\
& =0
\end{aligned}
$$

Since the bilinear form can be derived from the quadratic function it follows that the inner product of any two vectors in $\Gamma$ is integral. Similarly, we can show it is unimodular as follows: Suppose $(u ; v) \in \Gamma^{\vee}$. Then for all $(s ; t) \in \Gamma$ we have $-u \cdot s+v \cdot t=0 \bmod 1$. So, taking $t=0$ and then $s=0$ shows that $(u ; v) \in \tilde{\Gamma}$. we have

$$
-\bar{u} \cdot \bar{s}+\bar{v} \cdot \bar{t}=0 \bmod 1
$$

and hence

$$
\left(\bar{u}-\psi^{-1}(\bar{v})\right) \cdot \bar{s}=0 \bmod 1 .
$$

Now, since $\mathcal{D}_{+}\left(\mathfrak{F}_{L}^{\perp}\right)$ is a non-degenerate quadratic space we must have $\bar{u}-\psi^{-1}(\bar{v})=0$ and hence $\psi(\bar{u})=\bar{v}$ and hence $(u, v) \in \Gamma$.

Choosing an isomorphism $W_{d} \cong \mathbb{Q}^{24-d ; 8-d}$ and extending scalars to $\mathbb{R}$ we have constructed an embedding of $I I^{24-d ; 8-d}$ into $\mathbb{R}^{24-d ; 8-d}$.

Now, let $G_{L}=\operatorname{Fix}\left(\mathfrak{F}_{L}\right) \subset \operatorname{Aut}(\Lambda)=\mathrm{Co}_{0}$ and similarly $G_{R}$. Extending scalars we can embed $G_{L} \subset O(\Lambda \otimes \mathbb{Q}) \subset O(\Lambda \otimes \mathbb{R}) \cong O(24)$, and similarly $G_{R}$. Now we claim that if $x \in\left(\mathfrak{F}_{L}^{\perp}\right)^{\vee} \subset \Lambda \otimes \mathbb{Q}$ and $g \in G_{L}$ then

$$
g \cdot x-x \in \mathfrak{F}_{L}^{\perp}
$$

(a priori we only know that $g \cdot x-x \in\left(\mathfrak{F}_{L}^{\perp}\right)^{\vee}$ ). This follows since for any $x \in\left(\mathfrak{F}_{L}^{\perp}\right)^{\vee}$ there is an $x^{\prime} \in \mathfrak{F}_{L}^{\vee}$ so that $x \oplus x^{\prime} \in \Lambda$. But then

$$
g \cdot x-x=g \cdot\left(x \oplus x^{\prime}\right)-\left(x \oplus x^{\prime}\right) \in \Lambda
$$


But now (2.23) implies that in the discriminant group $g(\bar{x})=\bar{x}$ for any $x \in\left(\mathfrak{F}_{L}^{\perp}\right)^{\vee}$ and $g \in G_{L}$. Entirely similar remarks apply to $g \in G_{R}$ and $y \in\left(\mathfrak{F}_{R}^{\perp}\right)^{\vee}$.

Now we define the action of $G_{L} \times G_{R}$ on $\tilde{\Gamma}$ by

$$
\left(g_{L}, g_{R}\right) \cdot(x ; y):=\left(g_{L} \cdot x ; g_{R} \cdot y\right)
$$

Moreover, as we have seen $g_{L} \bar{x}=\bar{x}$ and $g_{R} \bar{y}=\bar{y}$. Therefore if $(x ; y) \in \Gamma$ then

$$
\psi\left(g_{L} \bar{x}\right)=\psi(\bar{x})=\psi(\bar{y})=\psi\left(g_{R} \bar{y}\right)
$$

and hence the action of $G_{L} \times G_{R}$ preserves $\Gamma$. This concludes the proof

In essence the lattice $\Gamma$ is simply given by adding suitable glue vectors to $\mathfrak{F}_{L}^{\perp} \oplus \mathfrak{F}_{R}^{\perp}+\cdots$. Given the above Lemma we now define the Conway subgroup symmetric compactifications of the heterotic string to be defined by those points in Narain moduli space associated with such pairs of isometric sublattices $\mathfrak{F}_{L} \subset \Lambda$ and $\mathfrak{F}_{R} \subset \Gamma_{8}$. We refer to these as CSS compactifications for short.

\section{Remarks:}

1. Höhn and Mason have tabulated the 290 isomorphism classes of sublattices $\mathfrak{L}_{L} \subset \Lambda$ such that the subgroup $\operatorname{Fix}\left(\mathfrak{F}_{L}\right) \subset \mathrm{Co}_{0}$ fixing all vectors of $\mathfrak{F}_{L}$ is nontrivial [43]. The reader of this paper who wants to follow the details of the analysis will find it useful to have Table 1 and supplementary Table 2 of [43] handy. Said reader might also note the versions two and three of [43] are useful for different purposes. We will refer to entries with number \# in their table as HM\# for short.

2. For $d=4$ there is a kind of converse of this result due to Gaberdiel, Hohenegger, and Volpato [31]. They have shown that $G \subset O_{\mathbb{Z}}\left(I I^{20,4}\right)$ fixes a positive definite four-dimensional subspace of $\mathbb{R}^{20,4}=I I^{20,4} \otimes \mathbb{R}$ iff $G$ is a subgroup of $\mathrm{Co}_{0}$ fixing a sublattice of $\Lambda$ of rank at least four. Although we will not use this result directly, it was important to our thinking.

\section{Examples Of CSS Compactifications}

We now turn to compactifications of the heterotic string on $T^{2} \times K 3$ preserving fourdimensional $N=2$ supersymmetry. These are notable for having type IIA dual compactifications on $K 3$-fibered Calabi-Yau threefolds ${ }^{5}$. (For a review, see [3].) We would like to consider such compactifications with Conway subgroup symmetry.

\footnotetext{
${ }^{5}$ We are not aware of any general proof that all heterotic compactifications with $N=2$ supersymmetry in four dimensions have type II duals when one includes the kind of non-geometrical compactifications used here involving asymmetric orbifold constructions
} 


\subsection{Right-moving Lattice $\mathfrak{F}_{R}$ And Orbifold Action On Right-Movers}

The natural way to produce CSS compactifications of the heterotic string on $T^{2} \times K 3$ is to consider suitable orbifolds of CSS compactifications on $T^{6}$. Compactification on such tori is the case $d=2$ in (2.1). Therefore we can produce models by considering the rank two sublattices $\mathfrak{F}_{L} \subset \Lambda$ in Tables 1 and 2 of [43] and then searching for isometric primitively embedded sublattices $\mathfrak{F}_{R} \subset \Gamma_{8}$ such that there is a subgroup $G_{R} \subset \operatorname{Fix}\left(\mathfrak{F}_{R}\right) \subset W\left(E_{8}\right)$ so that if $T^{6}=\mathbb{R}^{6} / \mathfrak{F} \frac{\perp}{R}$ then

$$
T^{6} / G_{R} \cong T^{2} \times S
$$

with $S$ an orbifold limit of a K3 surface.

For simplicity we will limit attention here to the simplest case of $G_{R} \cong \mathbb{Z}_{2}$. We therefore seek an involution in the Weyl group of $E_{8}$. Moreover, our "K3 surface" will be the orbifold $T^{4} / \mathbb{Z}_{2}$ and hence we need an involution with precisely four -1 eigenvalues, that is, one such that the character in the natural eight-dimensional representation is zero. There are two conjugacy classes of such involutions. We can describe one representative of the first conjugacy class as a sign flip on the first four coordinates (using the standard model for $\Gamma_{8}$ mentioned near (2.5)):

$$
\sigma_{1}=\left(-1^{4},+1^{4}\right) .
$$

A representative of the second conjugacy class acts on the coordinates as a matrix:

$$
\sigma_{2}=\left(\begin{array}{cc}
H & 0 \\
0 & H
\end{array}\right)
$$

where $H$ is the famous Hadamard matrix

$$
H:=\frac{1}{2}\left(\begin{array}{cccc}
1 & 1 & 1 & 1 \\
1 & -1 & 1 & -1 \\
1 & 1 & -1 & -1 \\
1 & -1 & -1 & 1
\end{array}\right) .
$$

It will be useful later to note that the four vectors $u_{i} \in \Gamma_{8}$ given by

$$
\begin{aligned}
& u_{1}=(1 / 2)(1,1,1,-1,1,1,1,-1) \\
& u_{2}=(1 / 2)(1,1,1,-1,-1,-1,-1,1) \\
& u_{3}=(1,0,0,1,0,0,0,0) \\
& u_{4}=(0,0,0,0,1,0,0,1)
\end{aligned}
$$

form a basis for the rank 4 sublattice of $\Gamma_{8}$ which is fixed by the Hadamard involution.

It is now a simple, albeit tedious matter to enumerate the possible rank two sublattices $\mathfrak{F}_{R} \subset \Gamma_{8}$ fixed by the above two involutions and compare with the sublattices $\mathfrak{F}_{L}$ in the tables of 43]. Of the $51 \mathrm{rank}$ two HM classes we find all but 6 . The missing classes are \#223, \#227, \#232, \#237, \#240, \#246. with Gram matrices:

$$
\left(\begin{array}{ll}
4 & 1 \\
1 & 4
\end{array}\right),\left(\begin{array}{ll}
4 & 1 \\
1 & 6
\end{array}\right),\left(\begin{array}{ll}
4 & 2 \\
2 & 8
\end{array}\right),\left(\begin{array}{cc}
4 & 2 \\
2 & 16
\end{array}\right),\left(\begin{array}{cc}
6 & 3 \\
3 & 12
\end{array}\right),\left(\begin{array}{ll}
8 & 2 \\
2 & 8
\end{array}\right),
$$


respectively. It is possible that these missing classes can be captured by considering other orbifold limits of K3 surfaces, but we have not investigated this possibility.

We also need to construct a primitive embedding of a sublattice $\mathfrak{F}_{R}$ into $\Gamma_{8}$ where $\mathfrak{F}_{R}$ has the desired Gram matrix. The existence of such an embedding is guaranteed by Theorem 1.12.2 of [62] and in explicit examples discussed below we will construct these primitive embeddings explicitly.

However there is no guarantee that such a primitive embedding can be chosen to lie within the invariant subspace of one of the involutions $\sigma_{1}, \sigma_{2}$. For the Gram matrix $Q^{224}$ in the following subsection there are obvious choices of orthogonal square-length four vectors fixed by the involution $\sigma_{1}$, but one can check that no such pair generates a primitive sublattice of $\Gamma_{8}$. In fact for both of the examples in the following subsection we can find a primitive embedding and choose basis vectors for $\mathfrak{F}_{R}$ that are in the $\sigma_{2}$-invariant subspace.

\subsection{Left-moving Lattice $\mathfrak{F}_{L}$}

We now focus on two illustrative examples chosen to have large CSS groups as stabilizers. The Gram matrices are \#222 and \#224 in [43] and are given by

$$
Q^{222}=\left(\begin{array}{cc}
4 & -2 \\
-2 & 4
\end{array}\right) \quad Q^{224}=\left(\begin{array}{ll}
4 & 0 \\
0 & 4
\end{array}\right) .
$$

According to Table 1 of 443 the group $\operatorname{Fix}\left(\mathfrak{F}_{L}\right)$ with Gram matrix $Q^{222}$ is a subgroup of $\mathrm{Co}_{0}$ isomorphic to $U_{6}(2)$. For definiteness, we will choose $\mathfrak{F}_{L}$ to be the sublattice of the Leech lattice generated by the vectors $v_{1}$ and $-v_{3}$ of (B.3).

Similarly, for $\mathfrak{F}_{L}$ with Gram matrix $Q^{224}$ Table 1 of 43 says the stabilizer group $\operatorname{Fix}\left(\mathfrak{F}_{L}\right) \cong 2^{10} \cdot M_{22}$, and moreover it is a subgroup of the monomial subgroup $2^{12}: M_{24}$ of the Leech lattice, described in Appendix B. As described in Appendix B we are using a presentation of the Leech lattice so that $\{1, \ldots, 8\}$ is a set in the Steiner system $S(5,8,24)$. For definiteness, we choose $\mathfrak{F}_{L}$ to be the sublattice generated by the vectors $v_{1}$ and $v_{2}$ of (B.3).

See Appendices $\mathrm{C}$ and $\mathrm{D}$ for the detailed construction of the entire Narain lattice $\Gamma$ of signature $(22,6)$ for each of these two choices of $\mathfrak{F}_{L}$.

\subsection{Orbifold Action On Left-Movers}

Once one has constructed $\Gamma$ and chosen the action of the involution on the right-moving coordinates it remains to choose the involution on the left-moving coordinates. This will be of the form

$$
x \rightarrow g_{L} x+\delta
$$

where $g_{L} \in \operatorname{Fix}\left(\mathfrak{F}_{L}\right)$ is an involution and $\delta$ is in $\Lambda \otimes \mathbb{Q}$ such that $g_{L} \delta+\delta \in \Lambda$. There are four conjugacy classes of involutions in $\mathrm{Co}_{0}$ with characters $-24,8,-8,0$ in the 24-dimensional irreducible representation. Thus in a diagonal basis $g_{L}$ will have $24,8,16,12$ eigenvalues -1 , respectively. Since we must have at least 4 eigenvalues +1 , to produce a compactification on $T^{2} \times K 3$, (these account for the $T^{2}$ and the $\mathbb{R}^{2}$ directions) the involution $g_{L}$ must be in one of the conjugacy classes with 8,16 , or 12 eigenvalues equal to -1 . 
We now define three concrete models corresponding to three choices of $\left(g_{L}, \delta\right)$. See Appendix $\mathrm{B}$ for the definitions of $\mathcal{C}$-set, octad and dodecad.

1. A: $g_{L}$ is a sign-flip on a $\mathcal{C}$-set fixing $\mathfrak{F}_{L}$. For both $Q^{222}$ and $Q^{224}$ we can choose an octad so that that sign flip fixes $x_{1}, x_{2}, x_{3}$. For example the flips associated with $S_{22}$ or $S_{23}$ will do. Moreover we take the shift vector to be $\delta=0$. Note that there is a variant of this model with a shift vector $\delta=\frac{1}{2} v_{4}$ with $v_{4} \in \Lambda$ with $v_{4}^{2}=4$, but we will analyze only the model with vanishing shift vector.

2. $B: g_{L}$ is a sign-flip on an octad-complement of a $\mathcal{C}$-set that leaves $\mathfrak{F}_{L}$ pointwise fixed. For example, a sign flip on the complement of $S_{8}$ will fix coordinates $x_{1}, x_{2}, x_{3}$ and hence will fix $\mathfrak{F}_{L}$ for both cases $Q^{222}$ and $Q^{224}$.

3. $C: g_{L}$ is a sign-flip on a dodecad $\mathcal{C}$-set that leaves $x_{1}, x_{2}$ invariant. To be concrete for $Q^{224}$ we choose the dodecad in $(\overline{B .5})$ so:

$$
\begin{array}{lr}
g_{L}^{C} x_{i}=x_{i} & i \in\{1,2,5,7,10,12,13,14,17,18,22,24\} \\
g_{L}^{C} x_{i}=-x_{i} & i \in\{3,4,6,8,9,11,15,16,19,20,21,23\}
\end{array}
$$

and for $Q^{222}$ we choose the dodecad in (B.5)

$$
\begin{aligned}
& g_{L}^{C} x_{i}=x_{i} \quad i \in\{1,2,3,4,9,10,15,16,19,20,23,24\} \\
& g_{L}^{C} x_{i}=-x_{i} \quad i \in\{5,6,7,8,11,12,13,14,17,18,21,22\}
\end{aligned}
$$

Level matching requires that we choose $\delta=\frac{1}{2} v$ with $v^{2}=2 \bmod 4$. In order to preserve a large crystallographic symmetry acting on the left we choose $v$ to be in the right-moving part of the lattice and invariant under the Hadamard involution. Thus we can choose $v$ to be any of the $u_{i}$ in (3.5).

The orbifold models $A, B, C$ all satisfy standard level-matching constraints required for modular invariance. In addition one should address the "DTF" criterion of [41] (which extends earlier treatments in [55, 60, 61].) Namely if there is a vector $p \in \Gamma$ such that

$$
(p, g \cdot p)=1 \bmod 2 .
$$

then, as described under equation (2.17) of [41] either $\hat{g}|p\rangle \neq|g \cdot p\rangle$ for some $p \in \Gamma^{g}$ or the lifted automorphism $\hat{g}$ on the CFT will be order four. When the DTF criterion (3.11) holds we will use the canonical lift of equations (6.35) and (6.36) of [41]. After some work one can show that for the involutions of type $A$ and $B$ the DTF condition (3.11) does indeed hold for involutions of type A,B for both $Q^{222}$ and $Q^{224}$. As a result models A,B are actually $\mathbb{Z}_{4}$ orbifolds. Strangely, for $C$ the condition (3.11) again holds for $Q^{222}$ but not for $Q^{224}$, so only in the last case do we have a true $\mathbb{Z}_{2}$ orbifold. Altogether we will analyze six $K 3 \times T^{2}$ orbifolds of CSS compactifications labelled by the two choices of Gram matrix (\#222 and \#224) and three choices of left-moving lattice involution $(A, B, C)$. 


\subsection{Massless States In The Untwisted Sector}

It is straightforward to compute the untwisted sector massless spectrum of these models. (For some details see Appendix A.) Let $n_{-}=8,16,12$ be the number of -1 eigenvalues of $g_{L}$ in cases $A, B, C$, respectively. In the untwisted sector one finds one $N=2$ supergravity multiplet, $23-n_{-} U(1)$ vectormultiplets, and $n_{-}$hypermultiplets. The massless scalars are moduli. Before dividing by duality symmetries we have a vector-multiplet moduli space

$$
\mathcal{M}_{v m}=\frac{S L(2, \mathbb{R})}{S O(2)} \times \frac{O\left(2,22-n_{-}\right)}{O(2) \times O\left(22-n_{-}\right)}
$$

and a hypermultiplet moduli space

$$
\mathcal{M}_{h m}=\frac{O\left(4, n_{-}\right)}{O(4) \times O\left(n_{-}\right)}
$$

The computation of the spectrum in the twisted sector for model $\mathrm{C}$ is also straightforward for the simple reason that the ground state energy in the twisted sector is positive so there are no massless twisted sector states and hence the full massless spectrum consists of 11 vectormultiplets and 12 hypermultiplets.

\subsection{Massless States In The Twisted Sectors}

For models $A, B$ with order four lifts there are massless states in the twisted sectors and we need to work much harder. To compute the massless twisted sector we begin with the trace in the unprojected theory: $\operatorname{Tr}_{\mathcal{H}} \hat{g} q^{L_{0}-c / 24} \bar{q}^{\tilde{L}_{0}-\tilde{c} / 24}$ where for the canonical lift we have

$$
\langle p|\hat{g}| p\rangle= \begin{cases}1 & p \in \Gamma^{g} \\ 0 & \text { else }\end{cases}
$$

Now let $Z\left(\hat{g}^{x}, \epsilon_{1} ; \hat{g}^{y}, \epsilon_{2}\right)$ be the twisted partition function with spin structure specified by $\left(\epsilon_{1}, \epsilon_{2}\right)$ (relative to the canonical RR spin structure) and twist $\hat{g}^{y}$ in the space and $\hat{g}^{x}$ in the time direction. Including orbifold and GSO projection the $\hat{g}$-twisted NS sector partition function is:

$$
Z\left(\mathcal{H}_{\hat{g}}\right)=\frac{1}{2} \cdot \frac{1}{4}\left[\sum_{x=0}^{3} \sum_{\epsilon= \pm} Z\left(\hat{g}^{x}, \epsilon ; \hat{g},-\right)\right] .
$$

Now, let the number of - eigenvalues be $n_{-}$on left-movers and $\tilde{n}_{-}$on right-movers. For our example $\tilde{n}_{-}=4$ but we will leave the formulae general for a little while, merely assuming $\tilde{n}_{-}>0$. Then the spatial twist $\hat{g},-$ means that there are $\tilde{n}_{-}$real fermions with periodic boundary conditions. Therefore $Z(\hat{g},-; \hat{g},-)$ and all its images under $\tau \rightarrow \tau+1$ vanish. This leaves four terms:

$$
Z\left(\mathcal{H}_{\hat{g}}\right)=\frac{1}{2} \cdot \frac{1}{4}\left[Z(1,-; \hat{g},-)+Z(\hat{g},+; \hat{g},-)+Z\left(\hat{g}^{2},-; \hat{g},-\right)+Z\left(\hat{g}^{3},+; \hat{g},-\right)\right]
$$

These are all $\tau \rightarrow \tau+1$ images of each other so if we are interested in the massless states, corresponding to the coefficient of $q^{0} \bar{q}^{0}$ we can say this is just

$$
\left.Z\left(\mathcal{H}_{\hat{g}}\right)\right|_{\text {massless }}=\frac{1}{2}[Z(1,-; \hat{g},-)]_{q^{0} \bar{q}^{0}}
$$


Now, because models A and B violate the DTF condition (3.11) the characteristic vector $W_{g}$ of 41] is nonzero. Since in the $\hat{g}^{2}$ twisted sector the momentum is shifted by $W_{g}$ and since the oscillators are untwisted that means the ground state must be massive. Thankfully, we don't have to compute the $\hat{g}^{2}$-twisted sector partition function for massless states. Moreover, the $\hat{g}^{3}=\hat{g}^{-1}$ sector should give a second copy of the massless states from the $\hat{g}$-twisted sector. Therefore, we conclude that the number of massless twisted states is just:

$$
\# \text { massless twisted real scalars }=[Z(1,-; \hat{g},-)]_{q^{0} \bar{q}^{0}}
$$

Now, it is easy to show that

$$
Z(1,-; \hat{g},-)=\left|\mathcal{D}\left(\Gamma^{g}\right)\right|^{-1 / 2} 2^{\left(n_{-}+\tilde{n}_{-}\right) / 2} F_{L}(\tau) \overline{F_{R}(\tau)} \Theta_{\left(\Gamma^{g}\right) \vee}(\tau)
$$

where $\Theta$ is the theta function without shift vector (following the conventions of [41]), while

$$
\begin{gathered}
F_{L}(\tau):=\left(\frac{1}{\eta(\tau)}\right)^{24-n_{-}}\left(\frac{\eta(\tau)}{\eta(\tau / 2)}\right)^{n_{-}} \\
F_{R}(\tau)=\left(\frac{1}{\eta(\tau)}\right)^{8-\tilde{n}_{-}}\left(\frac{\vartheta_{3}}{\eta}\right)^{4-\tilde{n}_{-} / 2}\left(\frac{\eta(\tau)}{\eta(\tau / 2)}\right)^{\tilde{n}_{-}}\left(\frac{\vartheta_{2}}{\eta(\tau)}\right)^{\tilde{n}_{-} / 2}
\end{gathered}
$$

In particular we have the leading $q$-expansions:

$$
\begin{gathered}
F_{L}=q^{-1+\frac{n_{-}}{16}} \frac{1}{\left(1-q^{1 / 2}\right)^{n_{-}}}[1+\mathcal{O}(q)] \\
F_{R}=2^{\tilde{n}_{-} / 2} q^{-\frac{1}{2}+\frac{\tilde{n}_{-}}{8}}\left[1+\mathcal{O}\left(q^{1 / 2}\right)\right]
\end{gathered}
$$

Let us stress that our partition function is in the NS sector. So (3.18) counts the number of real massless bosonic fields. They will form hypermultiplets with fermions from the $\mathrm{R}$ sector. If we let $T W$ denote the number of massless twisted real scalars then altogether the number of massless hypermultiplets is

$$
\# H M=\frac{1}{4} T W+n_{-}
$$

Now for model B we have $n_{-}=16$ and $\tilde{n}_{-}=4$. So $F_{L}$ starts at $q^{0}$ and $F_{R}$ starts at $\bar{q}^{0}$ so there no contributions from the lattice theta function. So

$$
\begin{aligned}
T W:=\# \text { massless twisted real scalars for model } \mathrm{B} & =\left|\mathcal{D}\left(\Gamma^{g_{B}}\right)\right|^{-1 / 2} 2^{\left(n_{-}+\tilde{n}_{-}\right) / 2} 2^{\tilde{n}_{-} / 2} \\
& =2^{12}\left|\mathcal{D}\left(\Gamma^{g_{B}}\right)\right|^{-1 / 2}
\end{aligned}
$$

Using the method described below in section 3.7 we find $\left|\mathcal{D}\left(\Gamma^{g_{B}}\right)\right|=2^{8}$ for both cases $Q^{222}$ and $Q^{224}$ and hence, plugging in the numbers there are 7 vectormultiplets and 80 hypermultiplets for both choices of $\mathfrak{F}_{L}$. Given the very different symmetry groups in these 
two cases we expect the dual Calabi-Yau threefolds to be very different in spite of their common Hodge numbers.

Similarly, for model A we have $n_{-}=8$ so

$$
F_{L}=q^{-1 / 2}\left(1+8 q^{1 / 2}+\mathcal{O}(q)\right)
$$

Now we can get a possible contribution from the theta function. Let $\mathcal{N}_{1,0}$ be the set of vectors $p$ in $\left(\Gamma^{g}\right)^{\vee}$ with $p_{L}^{2}=1$ and $p_{R}^{2}=0$. Then

$T W:=$ \#massless twisted real scalars for model $\mathrm{A}=\left|\mathcal{D}\left(\Gamma^{g_{A}}\right)\right|^{-1 / 2} 2^{\left(n_{-}+\tilde{n}_{-}\right) / 2} 2^{\tilde{n}_{-} / 2}\left(8+\left|\mathcal{N}_{1,0}\right|\right)$

$$
=2^{8}\left|\mathcal{D}\left(\Gamma^{g_{A}}\right)\right|^{-1 / 2}\left(8+\left|\mathcal{N}_{1,0}\right|\right)
$$

Using the method described below in section 3.7 we find $\left|\mathcal{D}\left(\Gamma^{g_{A}}\right)\right|=2^{12}$ for $Q^{222}$ and $\left|\mathcal{D}\left(\Gamma^{g_{A}}\right)\right|=2^{10}$ for $Q^{224}$. Moreover $\left|\mathcal{N}_{1,0}\right|=72$ for $Q^{222}$ and $\left|\mathcal{N}_{1,0}\right|=28$ for $Q^{224}$. Hence, plugging in the numbers there are 15 vectormultiplets in both cases and 88 hypermultiplets for $Q^{222}$ and 80 hypermultiplets for $Q^{224}$.

\subsection{The Fate Of Massless Charged Hypermultiplets}

For applications to heterotic/type II duality we must work a little harder in the case of $Q^{222}$ and $Q^{224}$. Indeed the numbers 88 and 80 of massless hypermultiplets is a little misleading because some of those hypermultiplets (those corresponding to states with $p \in \mathcal{N}_{1,0}$ ) will be charged under the $16 U(1)$ gauge symmetries. If there is a type II dual of the heterotic theory with zero vacuum expectation value for these charged scalar fields then we would expect the Calabi-Yau threefold to be somewhat singular. As in [36, 45], we expect that if the charged scalars take generic vacuum expectation values then the dual Calabi-Yau manifold will be smooth(er).

String perturbation theory should produce some potential energy in the Lagrangian for these scalars consistent with the general structure of $d=4, N=2$ supergravity. (See [29] chapters 20 and 21, or [21], for the general form such a potential energy function can take.) If the massless charged hypers get vacuum expectation values they will Higgs some collection of those left-moving $U(1)$ gauge bosons corresponding to the states listed in (A.3) below. We will assume that the potential for these charged scalar fields is sufficiently generic that all of the modes of the charged scalars that are not Goldstone bosons and that can become massive in a way consistent with $N=2$ supersymmetry, will become massive. Of course, the Goldstone bosons get eaten. ${ }^{6}$ Moreover, we will assume the vacuum is at a generic point in the moduli space of vacua and that the situation is sufficiently generic that the number of broken generators of the $U(1)^{24-n_{-}}$gauge group is the number, $\mathcal{L}_{1,0}$, of linearly independent vectors in $\mathcal{N}_{1,0}$. Of course, such vacua are outside the moduli space of toroidal orbifolds.

\footnotetext{
${ }^{6}$ In principle this assumption could be checked by computing the correlation functions of the vertex operators of the twisted charged massless scalar fields. Such a computation goes way beyond the scope of this paper and we will simply assume the potential energy generated by conformal perturbation theory is generic.
} 
Given the above genericity assumptions we can say that, after Higgsing, the number of vectormultiplets and massless neutral hypermultiplets is altered, for model A, to

$$
\begin{aligned}
& \text { \#vectormultiplets }=23-n_{-}-\mathcal{L}_{1,0}=15-\mathcal{L}_{1,0} \\
& \text { \#hypermultiplets }=n_{-}+2^{9}\left|\mathcal{D}\left(\Gamma^{g_{A}}\right)\right|^{-1 / 2}+\frac{1}{4} \operatorname{dim}_{\mathbb{R}} \mathcal{M}_{\text {Higgs }}
\end{aligned}
$$

To compute the dimension of the Higgs branch we proceed naively and count degrees of freedom and subtract the numbers of equations and symmetries to get ${ }^{7}$

$$
\operatorname{dim}_{\mathbb{R}} \mathcal{M}_{\text {Higgs }}=\frac{2^{8} \cdot\left|\mathcal{N}_{1,0}\right|}{\left|\mathcal{D}\left(\Gamma^{g_{A}}\right)\right|^{1 / 2}}-4 \mathcal{L}_{1,0}= \begin{cases}4 \cdot 60 & Q^{222} \\ 4 \cdot 42 & Q^{224}\end{cases}
$$

and hence

$$
\# \text { massless neutral hypermultiplets }= \begin{cases}76 & Q^{222} \\ 66 & Q^{224}\end{cases}
$$

\subsection{Computation Of Discriminant Groups, $\mathcal{N}_{1,0}$ And $\mathcal{L}_{1,0}$}

Clearly, to proceed we need to be able to compute the lattice $\left(\Gamma^{g}\right)^{\vee}$ both for the order of the discriminant group $\mathcal{D}\left(\Gamma^{g}\right)$ and in order to compute the vectors enumerated by $\mathcal{N}_{1,0}$. This is not at all easy, and is probably out of reach using purely human computational methods. We proceed using the following method which can be easily implemented using a (sufficiently bright) computer algebra system. Let $g$ be an automorphism of order two of a lattice $\Gamma$ and let $\Gamma^{g}$ be the sublattice of $\Gamma$ consisting of vectors in $\Gamma$ which are invariant under the action of $g$. It is not easy to compute $\Gamma^{g}$ directly, but given an explicit action of $g$ we can easily compute the lattice $(1+g) \Gamma$ consisting of vectors $(1+g) v$ with $v \in \Gamma$ as well as the lattice $P_{g} \Gamma$ with $P_{g}=(1+g) / 2$. The lattice $\Gamma^{g}$ is an overlattice of $(1+g) \Gamma$, meaning that $(1+g) \Gamma$ and $\Gamma^{g}$ have the same rank and that there is an embedding $(1+g) \Gamma \hookrightarrow \Gamma^{g}$. This is clear since every vector in $(1+g) \Gamma$ is invariant under $g$ and hence in $\Gamma^{g}$. However there might be vectors in $\Gamma^{g}$ which are not in $(1+g) \Gamma$. Similarly $P_{g} \Gamma$ is an overlattice of $\Gamma^{g}$, so we have a lattice sandwich:

$$
(1+g) \Gamma \subset \Gamma^{g} \subset \frac{1}{2}(1+g) \Gamma .
$$

It might be useful to keep two simple and illustrative examples in mind.

\footnotetext{
${ }^{7}$ To do this more properly one should use equations (21.16) and (21.30) of [29]. Again this goes beyond the scope of this paper. As a naive guess we expect that the $U(1)$ symmetry of the graviphoton remains unbroken so that $N=2$ supersymmetry is unbroken. (If this assumption were false it would be quite interesting.) Moreover, at weak string coupling one expects that the much simpler hyperkähler quotient construction appropriate to field theory can be used. (Certainly, this was assumed in [36, 45].) Since $\mathcal{L}_{1,0}<24-n_{-}$only a proper subgroup of the abelian gauge group can be Higgsed. We can write a finite cover of the gauge group in the form $U(1)^{\mathcal{L}_{1,0}} \times U(1)^{\mathrm{unb}}$ where $U(1)^{\mathrm{unb}}$ acts trivially on the scalars. Then we take the hyperkähler quotient with respect to $U(1)^{\mathcal{L}_{1,0}}$. This might leave an unbroken finite group gauge symmetry.
} 
Example 1. Let $\Gamma$ by the $A_{2}$ root lattice with simple roots $r_{1}, r_{2}$ and let $g$ be the reflection in the hyperplane orthogonal to $r_{1}$ so that $g: r_{1} \rightarrow-r_{1}, r_{2} \rightarrow r_{1}+r_{2}$. Vectors in $\Gamma^{g}$ are all multiples of $(1+g) r_{2}$. Thus in this example $\Gamma^{g}=(1+g) \Gamma=\mathbb{Z}\left(r_{1}+2 r_{2}\right)$ while $P_{g} \Gamma=\mathbb{Z}\left(\frac{1}{2} r_{1}+r_{2}\right)$.

Example 2. Let $\Gamma$ be the two-dimensional square lattice with basis vectors that are unit vectors $e_{x}, e_{y}$ and let $g$ be a reflection in the $y$ axis. Then $\Gamma^{g}=P_{g} \Gamma=\mathbb{Z} e_{y}$ while $(1+g) \Gamma=2 \mathbb{Z} e_{y}$.

To determine $\Gamma^{g}$ we now proceed as follows. Let $r$ be the rank of $\Gamma$ and $s \leq r$ the rank of $(1+g) \Gamma$. Let $b_{a}, a=1, \ldots, r$ be a basis for $\Gamma$ and $u_{j}, j=1, \ldots, s$ a basis for $(1+g) \Gamma$. A basis $u_{j}$ can be obtained by applying the LLL lattice basis reduction algorithm [54 to the matrix formed by the vectors $(1+g) b_{a}$. We can clearly express the $u_{j}$ as integer linear combinations of the $b_{a}$,

$$
u_{j}=\sum_{a} M_{j, a} b_{a}
$$

for some $M \in \operatorname{Mat}(s, r, \mathbb{Z})$. We can determine $M$ as follows. Taking the inner product on both sides with $b_{a^{\prime}}$ gives

$$
\left(u_{j}, b_{a^{\prime}}\right)=\sum_{a} M_{j, a}\left(b_{a}, b_{a^{\prime}}\right)=\sum_{a} M_{j, a} Q_{a, a^{\prime}}
$$

and since $Q_{a, a^{\prime}}=\left(b_{a}, b_{a^{\prime}}\right)$ is invertible we can just solve for $M$ as

$$
M_{j, a}=\left(u_{j}, b_{a^{\prime}}\right) Q_{a^{\prime}, a}^{-1}
$$

Next, $u_{j} / 2$ is a basis for $P_{g} \Gamma$ and any vector in $\Gamma^{g}$ is in $P_{g} \Gamma$. However not all vectors in $P_{g} \Gamma$ are in $\Gamma^{g}$ because not all of them are in $\Gamma$. A vector $\frac{1}{2} \sum_{j} n_{j} u_{j} \in P_{g} \Gamma$ is in $\Gamma^{g}$ precisely when it is in $\Gamma$, that is when $\frac{1}{2} \sum_{j, a} n_{j} M_{j, a} b_{a} \in \Gamma$, and this in turn is equivalent to the set of equations:

$$
\sum_{j} n_{j} M_{j, a}=0 \bmod 2 \quad \forall a=1, \ldots, r
$$

since elements of $\Gamma$ must be integer linear combinations of basis vectors. Taking all $n_{j}$ even always gives a solution. The nontrivial solutions, indicating that there are vectors in $\Gamma^{g}$ which are not in $(1+g) \Gamma$, arise when some of the $n_{j}$ are odd integers. To obtain $\Gamma^{g}$ we thus take the basis $u_{j}$ for $(1+g) \Gamma$ and construct an over complete set of vectors by adjoining vectors corresponding to all the nontrivial solutions of (3.35). This set of vectors forms an overcomplete set of vectors for $\Gamma^{g}$ and from this a basis can be constructed using the LLL algorithm [54]. Once one has a basis it is straightforward to compute the order of the discriminant group from the determinant of the Gram matrix.

One can similarly use the inverse Gram matrix to produce a basis for $\left(\Gamma^{g}\right)^{\vee}$ and then, with some more effort one can solve for the vectors $p$ with $p_{L}^{2}=1$ and $p_{R}^{2}=0$ and check how many such vectors are linearly independent. We provide some details and intermediate steps of this calculation for models based on $Q^{224}$ in Appendix D. 


\begin{tabular}{|c|c|c|c|}
\hline Höhn-Mason 222 & Model A & Model B & Model C \\
\hline signature $\left(\Gamma^{g}\right)$ & $(14,2)$ & $(6,2)$ & $(10,2)$ \\
\hline $\mathcal{D}\left(\mathfrak{F}_{L}\right)$ & $\mathbb{Z}_{2} \oplus \mathbb{Z}_{2} \oplus \mathbb{Z}_{3}$ & $\mathbb{Z}_{2} \oplus \mathbb{Z}_{2} \oplus \mathbb{Z}_{3}$ & $\mathbb{Z}_{2} \oplus \mathbb{Z}_{2} \oplus \mathbb{Z}_{3}$ \\
\hline Left-moving crystal symmetry & $\mathbb{Z}_{2}^{4} \cdot \mathbb{Z}_{2}^{4} \cdot A_{4} \cdot \mathrm{wr}_{2} \cdot \mathbb{Z}_{2}$ & $\mathbb{Z}_{2} \cdot \mathbb{Z}_{2}^{8} \cdot \operatorname{Sp}\left(4, \mathbb{Z}_{3}\right)$ & $\mathbb{Z}_{2} \times\left(\mathbb{Z}_{2}^{8} \cdot \mathbb{Z}_{3}^{2} \cdot Q_{8}\right)$ \\
\hline DTF? & Yes & Yes & Yes! \\
\hline$\left|\mathcal{D}\left(\Gamma^{g}\right)\right|$ & $2^{12}$ & $2^{8}$ & $\mathrm{x}$ \\
\hline$\left(\left|\mathcal{N}_{1,0}\right|, \mathcal{L}_{1,0}\right)$ & $(72,12)$ & $\mathrm{x}$ & $\mathrm{x}$ \\
\hline$(\#$ VM, \# HM $)$ & $(15,88)$ & $(7,80)$ & $(11,12)$ \\
\hline$\left(h^{1,1}, h^{2,1}\right)$ of hypothetical dual CY $\mathfrak{X}$ & $(3,75)$ & $(7,79)$ & $(11,11)$ \\
\hline
\end{tabular}

Table 1: Results for models A,B,C based on Gram matrix \#222 of [43].

\subsection{The Discrete Symmetries}

Finally, recall that we are particularly interested in these models because they have large and interesting discrete symmetries. These symmetries will include (a possible lift of) the left-moving crystal symmetry originating from the original Conway group symmetry. (This is the left-moving part of the group denoted $F(\Gamma)$ in [41.) As noted above, the symmetry group $\operatorname{Aut}\left(\mathfrak{F}_{L}^{\perp}\right) \subset C o_{0}$ (which is, in general, only a subgroup of the full group of automorphisms of $\mathfrak{F}_{L}^{\perp}$ ) is $G^{222}=U_{6}(2)$ and the subgroup of the monomial group $G^{224}=$ $2^{10} \cdot M_{22}$. Since we have chosen any shift vector to be right-moving we simply need to compute the centralizer of the involutions $g_{A}, g_{B}$ and $g_{C}$. The generators of $\operatorname{Aut}\left(\mathfrak{F}_{L}^{\perp}\right) \subset C o_{0}$ are provided in [43] and we can then use GAP and Magma to identify the conjugacy class of the involutions $g_{A}, g_{B}$, and $g_{C}$ and then compute their centralizers in $\operatorname{Aut}\left(\mathfrak{F}_{L}^{\perp}\right) \subset C o_{0}$. By studying the normal subgroups of the centralizer groups we obtain the descriptions of the groups given in Table 1 and Table 2 .

\subsection{Summary Of The Six Models}

We can summarize our results in two tables. The first table concerns HM222 and the second HM224.

In the above tables the notation " $\mathrm{x}$ " means we will not need to know the answer (or the entry is not applicable), while "wr" refers to a wreath product, $S p\left(4, \mathbb{Z}_{3}\right)$ is the symplectic group of rank two over the field $\mathbb{Z}_{3}$ and $Q_{8}$ is the quaternion group. Moreover, in writing the Hodge numbers for model $\mathrm{A}$ in the last line of the table we have assumed a generic potential giving mass to all the charged massless hypermultiplet scalars, as described in section 3.6 . 


\begin{tabular}{|c|c|c|c|}
\hline Höhn-Mason 224 & Model A & Model B & Model C \\
\hline signature $\left(\Gamma^{g}\right)$ & $(14,2)$ & $(6,2)$ & $(10,2)$ \\
\hline $\mathcal{D}\left(\mathfrak{F}_{L}\right)$ & $\mathbb{Z}_{4} \oplus \mathbb{Z}_{4}$ & $\mathbb{Z}_{4} \oplus \mathbb{Z}_{4}$ & $\mathbb{Z}_{4} \oplus \mathbb{Z}_{4}$ \\
\hline Left-moving crystal symmetry & $\mathbb{Z}_{2}^{8} .\left(\mathbb{Z}_{2} \times \mathbb{Z}_{2} \times D_{8}\right) \cdot G L_{2}\left(\mathbb{Z}_{7}\right)$ & $\mathbb{Z}_{2}^{10} \cdot\left(\mathbb{Z}_{2}^{4}: A_{6}\right)$ & $2^{x} \cdot M_{12}$ \\
\hline DTF? & Yes & Yes & No \\
\hline$\left|\mathcal{D}\left(\Gamma^{g}\right)\right|$ & $2^{10}$ & $2^{8}$ & $\mathrm{x}$ \\
\hline$\left(\left|\mathcal{N}_{1,0}\right|, \mathcal{L}_{1,0}\right)$ & $(28,14)$ & $\mathrm{x}$ & $\mathrm{x}$ \\
\hline$(\#$ VM, \# HM) & $(15,80)$ & $(7,80)$ & $(11,12)$ \\
\hline$\left(h^{1,1}, h^{2,1}\right)$ of hypothetical dual CY $\mathfrak{X}$ & $(1,65)$ & $(7,79)$ & $(11,11)$ \\
\hline
\end{tabular}

Table 2: Results for models A,B,C based on Gram matrix \#224 of [43].

\section{Implications Of Heterotic-Type II Duality}

Thanks to heterotic-type II duality the above results should have some implications for the type II string and for Calabi-Yau geometry. Our remarks in this section are rather more speculative than what we have discussed above. We hope they are positively provocative.

\subsection{Finding Type II Duals}

We begin with a few comments on the potential IIA Calabi-Yau 3-fold duals to the heterotic models discussed above. It is not, a priori obvious that heterotic/type II duality should apply to asymmetric orbifolds of heterotic string. Moreover, as far as we are aware, given a heterotic model on $T^{2} \times K 3$ with four-dimensional $N=2$ supersymmetry there is unfortunately no constructive procedure for identifying the dual type IIA Calabi-Yau 3 -fold. We will assume such a dual exists and make a few simple remarks concerning its properties.

The gauge symmetry is abelian and this limits the kinds of singularities the dual can have. Moreover, the Calabi-Yau should be K3-fibered, by the "adiabatic argument" of [67]. Moreover, the untwisted hypermultiplet moduli include moduli that one can use to give a six-dimensional limit where the size of the $T^{2}$ grows. (This follows from a detailed investigation of the lattice invariant under $\left(g_{R}, g_{L}\right)$.) Therefore, according to [3], $\mathfrak{X}$ should be elliptically fibered.

Assuming the holonomy of the dual $\mathfrak{X}$ to be generic the standard rule is that the Hodge numbers of $\mathfrak{X}$ are related to the four-dimensional $N=2$ supergravity by

$$
h^{1,1}(\mathfrak{X})=\# V M \quad h^{2,1}(\mathfrak{X})=\# H M-1
$$

Unfortunately there can be multiple Calabi-Yau's matching these Hodge numbers. For example for case $C$ we have $\left(h^{1,1}, h^{2,1}\right)=(11,11)$. These are the Hodge numbers of the famous Borcea-Voisin 3-fold used in the FHSV construction [28]. On the other hand, section 7.2.3 of [8] gives another construction of a CY 3 -fold $\mathfrak{X}$ with the same Hodge numbers and $\pi_{1}(\mathfrak{X})=\mathbb{Z}_{2}$. It is not obvious that these 3 -folds are equivalent. (See 24] for further relevant information.) Moreover, as we have seen with 222B and 224B, the discrete 
symmetries in the two examples are very different, even though the Hodge numbers are identical, suggesting that the hypothetical dual CY 3-folds must be different.

Using the tables for the Calabi-Yau Explorer of B. Jurke we can identify candidate CY 3 -folds with the expected Hodge numbers appearing in our tables above:

1. $(3,75)$ is discussed in several places including [35] [49].

2. $(7,79)$ is discussed in [9, 52, 53.

3. $(11,11)$ is discussed in $[8,24,28]$.

4. $(1,65)$ is discussed in [6, 35, 47].

One can learn a great deal about the potential dual Calabi-Yau by performing certain one-loop computations in the heterotic orbifold [1, 38, 42, 50, 57]. This includes the intersection numbers along with enumerative invariants associated with holomorphic curves in the Calabi-Yau. The intersection numbers are particularly useful for identifying the dual. To obtain these the relevant one-loop integral (for threshhold corrections of the gauge couplings $\left(\frac{1}{g^{2}}\right)^{a b}$ of the abelian gauge symmetries) takes the form

$$
\int_{\mathcal{F}} \frac{d^{2} \tau}{\tau_{2}}\left\{\sum_{I} F_{I}(\tau) \Theta_{\Gamma^{g}}\left(\alpha_{I}, \beta_{I} ;\left(p_{L}^{a} p_{L}^{b}-\frac{G^{a b}}{4 \pi \tau_{2}}\right)\right)-b_{\text {gauge }}^{a b}\right\} .
$$

Here $\mathcal{F}$ is a fundamental domain for $P S L(2, \mathbb{Z})$ acting on the $\tau$-upper half-plane, the sum on $I$ is a finite sum resulting from a sum over orbifold sectors and elements of the discriminant group of $\Gamma^{g}$, while $F_{I}(\tau)$ are simple modular forms for congruence subgroups, $\alpha_{I}, \beta_{I}$ are characteristics for a Siegel-Narain theta function with an insertion of $p_{L}^{a} p_{L}^{b}-\frac{G^{a b}}{4 \pi \tau_{2}}$, and finally $b_{\text {gauge }}^{a b}$ is the beta-function for the gauge couplings. The intersection numbers can be extracted from the "degenerate-orbit" contribution to the integral. The result is a concrete expression for the polynomial $d_{A B C} y^{A} y^{B} y^{C}$, where $d_{A B C}$ are the intersection numbers of the hypothetical Calabi-Yau and $y^{A}$ are special coordinates on the vectormultiplet moduli space (3.12). The expression that emerges is a sum of rational expressions in $y^{A}$ (and is not at all obviously a polynomial). The sum is obtained by choosing a finite-index embedding of $\Lambda_{0}=K \oplus I I^{2,2}(n)$ for some positive integer $n$ and some positive definite lattice $K$ into $\Gamma^{g}$. The resulting sum is then a finite sum over $I$, the elements of the quotient group $\Gamma^{g} / \Lambda_{0}$ and vectors in $K$ of norm-square two. We hope to present the details elsewhere.

\subsection{Auto-Equivalences Of The Derived Category}

The theorem of Gaberdiel, Hohenegger, and Volpato mentioned above was interpreted by Huybrechts in terms of the group of auto-equivalences of the derived category of a K3surface [4]. Identifying D-brane bound states with cohomology classes of moduli spaces of objects in the derived category, it is natural to interpret Huybrechts' theorem via heterotic type II duality. In particular, the list of relevant groups that can be identified as autoequivalences preserving the symplectic and stability structure is precisely the list of groups preserving rank four lattices in the Höhn-Mason tables. 
Given our orbifold CSS compactifications and heterotic-type II duality it is natural to speculate that there is a generalization of Huybrecht's theorem to Calabi-Yau pencils of $K 3$ surfaces. That is, we consider CY 3 -folds $\mathfrak{X}$ with $\pi: \mathfrak{X} \rightarrow \mathbb{P}^{1}$ such that the generic fibers of $\pi$ are smooth $K 3$ surfaces. In this case one can define a triangulated subcategory $D^{b}(\mathfrak{X})_{\text {vert }}$ of $D^{b}(\mathfrak{X})$, roughly speaking generated by coherent sheaves supported on the fibers of $\pi$, and it should be possible to define stability conditions $\sigma_{\text {vert }}$ on $D^{b}(\mathfrak{X})_{\text {vert }}$ compatible with a stability condition $\sigma$ on $D^{b}(\mathfrak{X})$ as defined in [4, 10]. For very general reasons the autoequivalences of $D^{b}(\mathfrak{X})$ preserving $\sigma$ form a finite group [11]. In view of heterotic/type II duality and our considerations regarding CSS compactifications it is natural to expect that autoequivalences of $D^{b}(\mathfrak{X})_{\text {vert }}$ preserving $\sigma_{\text {vert }}$ should be related to subgroups of the Conway group fixing sublattices of $\Lambda$ of rank two. In particular, we would predict that for duals to the models discussed in the previous sections the groups listed in the two tables (or perhaps extensions thereof) are the groups of autoequivalences. Of course, this is a highly nontrivial mathematical claim, one which might be quite challenging to confirm or disprove.

Clearly, these are only meant to be preliminary remarks, and we leave a fuller discussion of this idea to another occasion.

\subsection{Categories Of D-Branes}

The above considerations also raise two potential applications to categories of branes. ${ }^{8}$

To introduce the first question recall that there is a large mathematical literature interpreting the bound states of D-branes on Calabi-Yau manifolds $\mathfrak{X}$ in terms of triangulated categories with stability condition. For the $I I A$ string we find the derived category $D^{b}(\mathfrak{X})$ and for the $I I B$ string the Fukaya category $\operatorname{Fuk}(\mathfrak{X})$. Homological mirror symmetry identifies $D^{b}(\mathfrak{X})$ with $\operatorname{Fuk}(\mathfrak{Y})$ where $\mathfrak{Y}$ is the mirror of $\mathfrak{X}\left[51\right.$, $\mathbb{4}$. (More precisely it is an $A_{\infty}$ equivalence of $\operatorname{Fuk}(\mathfrak{Y})$ with an $A_{\infty}$ version of $D^{b}(\mathfrak{X})$.)

Our first question, is then: Is there a similar mathematical discussion of D-brane bound states on $S^{1} \times \mathfrak{X}$ ? Of course, the wrapped D-branes will either sit at a point in $S^{1}$ or wrap the $S^{1}$. So, for example, if $\mathfrak{X}$ is a 3 -fold then in the IIA string typical bound states will consist of $D 6-D 4-D 2-D 0$ bound states in $\mathfrak{X}$ localized at a point in $S^{1}$ and wrapping a holomorphic cycle in $\mathfrak{X}$. If we only consider standard Lagrangian branes in Fuk $(\mathfrak{X})$ then, without loss of generality, we can take the other brane to be a D4 wrapping $S^{1} \times L$ where $L$ is a Lagrangian subvariety of $\mathfrak{X}$. Simple considerations of the ground-state energies of open string states between these two kinds of branes suggest that they might form bound states. 9 Thus, mathematically one might ask if there exists an extension of the "union" of $D^{b}(\mathfrak{X})$ and $\operatorname{Fuk}(\mathfrak{X})$ that includes nontrivial morphisms between A- and B- branes. Since $S^{1} \times \mathfrak{X}$

\footnotetext{
${ }^{8}$ In both these remarks we are ignoring potential large IR gravitational effects of low-dimensional compactifications. We can try to avoid this by a $g_{\text {string }} \rightarrow 0$ limit, potentially limiting the applicability of these remarks to a "geometric engineering" subcategory of branes, such as that discussed in [15].

${ }^{9}$ Assuming the (possibly nontransverse) intersections are generic there will be 4 Neumann-Dirichlet type open strings in a formulation based on perturbative open string theory in the NSR formalism. As explained in Chapter 13, vol. 2 of [63] at leading order supersymmetry will be unbroken and the worldsheet ground state energy in the NS sector will be zero. However, since these configurations are in a curved Calabi-Yau manifold there can be perturbative corrections to the potential energy of deformation scalars and it is
} 
is neither complex nor symplectic one might at first think that topological field theory methods are completely unsuitable for investigating these issues but that is not the case: At the self-dual radius of $S^{1}$ there is an $N=3$ superalgebra with $\operatorname{Spin}(3) R$-symmetry [23] and so in fact one can define a variety of $A$ - and $B$-models and $A$ - and $B$-branes.

Our second question is motivated by the interpretation of CSS points in terms of type II moduli. Following the discussion of [2, 3] (see also Appendix C of [58]) it is clear that Conway subgroup symmetry of the D-brane system only arises when suitable flat RR fields are turned on. Our second question then is: How do flat RR fields affect bound states of Dbranes, and can these effects be incorporated into the above categorical description? As an extreme version of this we note that the type II dual to the distinguished compactification based on $\Gamma_{*}^{24 ; 8}$ must be a compactification on $K 3 \times T^{4}$ with suitable flat $B$-fields and flat $R R$-fields turned on. As an example of the odd things that can happen, if we replace the Leech lattice in $\Gamma_{*}^{24 ; 8}$ by the $E_{8}^{3}$ Niemeier lattice then the heterotic compactification should have a type II dual with $E_{8}^{3}$ gauge symmetry, something which seems rather exotic in the framework of F-theory.

Once again, these remarks are extremely preliminary, and we leave further investigation of these ideas for another time.

\section{A. Computation Of The Untwisted Sector For The Four-Dimensional CSS Models}

It is most convenient to work in the light-cone NSR formalism when deriving the massless spectrum of a $\mathbb{Z}_{2}$ orbifold with generator $\left(\left\{g_{L} ; \delta\right\}, g_{R}\right)$ where $\left\{g_{L} ; \delta\right\}$ is Seitz notation for the crystallographic symmetry $x \rightarrow g_{L} \cdot x+\delta$.

We divide up the indices of the oscillators as follows:

1. $\alpha_{n}^{\mu}, \mu=2,3$. (Left-moving) Oscillations in noncompact spacetime orthogonal to the light cone. They have $g_{L}=+1$.

2. $\alpha_{n}^{i}, i=1, \ldots, 22-n_{-}$. Oscillations in compact directions with $g_{L}=+1$.

3. $\alpha_{n}^{a}, a=1, \ldots, n_{-}$. Oscillations in compact directions with $g_{L}=-1$.

4. $\tilde{\psi}_{r}^{\mu}, \mu=2,3$. (Right-moving) Oscillations in noncompact spacetime orthogonal to the light cone. They have $g_{R}=+1$.

5. $\tilde{\psi}_{r}^{i}, i=4,5$. Oscillations in compact directions with $g_{R}=+1$.

6. $\tilde{\psi}_{r}^{a}, a=6,7,8,9$. Oscillations in compact directions with $g_{R}=-1$.

The only massless untwisted sector states have $P=0$. One might worry about states with $P=\left(p_{L} ; p_{R}\right)$ with $\frac{1}{2} p_{L}^{2}=+1$ and $\frac{1}{2} p_{R}^{2}=\frac{1}{2}$. But such vectors would not be even and hence don't exist. Therefore the only massless untwisted sector states have $P=0$. It is easy to list them:

entirely possible that these configurations sit at a local maximum in the potential energy. In such a case a small perturbation could lead to an instability that could settle on a nontrivial supersymmetric bound state. 
1. Metric, $B$-field, and dilaton:

$$
\alpha_{-1}^{\mu}|0\rangle \otimes \tilde{\psi}_{-1 / 2}^{\nu}|0\rangle
$$

Note that the dilaton and $B$-field are both real scalars.

2. Two abelian gauge fields from right-moving invariant subspace of $\mathbb{R}^{6,22}$ :

$$
\alpha_{-1}^{\mu}|0\rangle \otimes \tilde{\psi}_{-1 / 2}^{i}|0\rangle
$$

3. $22-n_{-}$abelian gauge fields from left-moving invariant subspace of $\mathbb{R}^{6,22}$ :

$$
\alpha_{-1}^{i}|0\rangle \otimes \tilde{\psi}_{-1 / 2}^{\nu}|0\rangle
$$

4. $2\left(22-n_{-}\right)$real scalar fields from the invariant subspaces of $\mathbb{R}^{6,22}$ :

$$
\alpha_{-1}^{i}|0\rangle \otimes \tilde{\psi}_{-1 / 2}^{j}|0\rangle
$$

5. $4 n_{-}$real scalar fields from the anti-invariant subspaces of $\mathbb{R}^{6,22}$ :

$$
\alpha_{-1}^{a}|0\rangle \otimes \tilde{\psi}_{-1 / 2}^{b}|0\rangle
$$

We get a total of 96 states - independent of $n_{-}$. This is confirmed by writing partition functions implementing the orbifold and GSO projections.

We can now organize these states into $d=4 N=2$ multiplets as follows: Two states from (A.1) correspond to the graviton. The other two are scalars. There are $24-n_{-}$vector fields. One of them is the graviphoton. Therefore there are $23-n_{-} U(1)$ vectormultiplets. Therefore, there are $2\left(23-n_{-}\right)=46-2 n_{-}$real vectormultiplet scalars. On the other hand, the number of scalars is

$$
T W+2+2\left(22-n_{-}\right)+4 n_{-}=T W+46+2 n_{-}
$$

where $T W$ is the number of real massless scalar fields from the twisted sectors. Subtracting the $46-2 n_{-}$vectormultiplet scalars we have

$$
T W+4 n_{-}
$$

hypermultiplet scalars, and therefore $\frac{1}{4} T W+n_{-}$hypermultiplets.

\section{B. The Binary Golay Code, The Steiner System $S(5,8,24)$, And The Leech Lattice}

We recall a few key definitions. The extended binary Golay code $\mathcal{G}$ is a 12 -dimensional subspace of $\mathbb{F}_{2}^{24}$. The vectors in $\mathcal{G}$, written as strings of 24 zeroes and ones, are called codewords. These codewords are divided up into 759 octads, consisting of codewords with 8 nonzero and 16 zero entries, 759 octad complements, 2576 dodecads, consisting of codewords with 12 nonzero entries and 12 zero entries, along with the origin $\left(0^{24}\right)$, and the 
codeword $\left(1^{24}\right)$. To any codeword we can assign a subset of the twentyfour element set $\Omega:=\{1, \ldots, 24\}$ using the positions of the nonzero coordinates. These subsets of $\Omega$ are known as $\mathcal{C}$-sets. Using the definition

$$
S_{1}+S_{2}:=\left(S_{1}-S_{2}\right) \amalg\left(S_{2}-S_{1}\right)
$$

the power set of $\Omega$ becomes a 24-dimensional vector space over $\mathbb{Z}_{2}$, thus recovering $\mathcal{G}$ from the set of $\mathcal{C}$-sets. The collection of 8 -element $\mathcal{C}$-sets - also referred to as octads - form a collection of 8 -element subsets of $\Omega$, known as the Steiner system $S(5,8,24)$. The Steiner system is distinguished by the property that any five element subset of $\Omega$ is a subset of a unique octad. The octads of the Golay code generate the entire space $\mathcal{G}$ using the operation + defined in (B.1). The Mathieu group $M_{24}$ is the group of permutations acting as automorphisms of $\mathcal{G}$, or, equivalently, as automorphisms of $S(5,8,24)$.

The Golay code can be used to give a concrete construction of the Leech lattice as a lattice embedded in $\mathbb{R}^{24}$ as follows. We take $\Lambda$ to be the set of vectors $x:=\left(x_{1}, \ldots, x_{24}\right)=$ $c\left(n_{1}, \ldots, n_{24}\right)$ where $c=1 / \sqrt{8}$, and $n_{i} \in 2 \mathbb{Z}+\epsilon$ with $\epsilon=0$ or $\epsilon=1$ for all $i$. We require moreover that $\sum_{i=1}^{24} n_{i}=4 \epsilon \bmod 8$, and finally we require that for each $x \in \Lambda$, each of the four sets:

$$
\mathcal{C}_{a}(x):=\left\{i \mid n_{i}=a \bmod 4\right\} \subset \Omega
$$

for $a=0,1,2,3$ is either the empty set or a $\mathcal{C}$-set in the Golay code derived from the Steiner system $S(5,8,24)$.

An explicit basis for the Leech lattice is 


$$
\begin{aligned}
v_{1} & =c\{4,-4,0,0,0,0,0,0,0,0,0,0,0,0,0,0,0,0,0,0,0,0,0,0\} \\
v_{2} & =c\{4,4,0,0,0,0,0,0,0,0,0,0,0,0,0,0,0,0,0,0,0,0,0,0\} \\
v_{3} & =c\{4,0,4,0,0,0,0,0,0,0,0,0,0,0,0,0,0,0,0,0,0,0,0,0\} \\
v_{4} & =c\{4,0,0,4,0,0,0,0,0,0,0,0,0,0,0,0,0,0,0,0,0,0,0,0\} \\
v_{5} & =c\{4,0,0,0,4,0,0,0,0,0,0,0,0,0,0,0,0,0,0,0,0,0,0,0\} \\
v_{6} & =c\{4,0,0,0,0,4,0,0,0,0,0,0,0,0,0,0,0,0,0,0,0,0,0,0\} \\
v_{7} & =c\{4,0,0,0,0,0,4,0,0,0,0,0,0,0,0,0,0,0,0,0,0,0,0,0\} \\
v_{8} & =c\{2,2,2,2,2,2,2,2,0,0,0,0,0,0,0,0,0,0,0,0,0,0,0,0\} \\
v_{9} & =c\{4,0,0,0,0,0,0,0,4,0,0,0,0,0,0,0,0,0,0,0,0,0,0,0\} \\
v_{10} & =c\{4,0,0,0,0,0,0,0,0,4,0,0,0,0,0,0,0,0,0,0,0,0,0,0\} \\
v_{11} & =c\{4,0,0,0,0,0,0,0,0,0,4,0,0,0,0,0,0,0,0,0,0,0,0,0\} \\
v_{12} & =c\{2,2,2,2,0,0,0,0,2,2,2,2,0,0,0,0,0,0,0,0,0,0,0,0\} \\
v_{13} & =c\{4,0,0,0,0,0,0,0,0,0,0,0,4,0,0,0,0,0,0,0,0,0,0,0\} \\
v_{14} & =c\{2,2,0,0,2,2,0,0,2,2,0,0,2,2,0,0,0,0,0,0,0,0,0,0\} \\
v_{15} & =c\{2,0,2,0,2,0,2,0,2,0,2,0,2,0,2,0,0,0,0,0,0,0,0,0\} \\
v_{16} & =c\{2,0,0,2,2,0,0,2,2,0,0,2,2,0,0,2,0,0,0,0,0,0,0,0\} \\
v_{17} & =c\{4,0,0,0,0,0,0,0,0,0,0,0,0,0,0,0,4,0,0,0,0,0,0,0\} \\
v_{18} & =c\{2,0,2,0,2,0,0,2,2,2,0,0,0,0,0,0,0,0,0,0,0,0,2,2\} \\
v_{19} & =c\{2,0,0,2,2,2,0,0,2,0,2,0,0,0,0,0,0,0,0,0,0,2,0,2\} \\
v_{20} & =c\{2,2,0,0,2,0,2,0,2,0,0,2,0,0,0,0,0,0,0,0,2,0,0,2\} \\
v_{21} & =c\{0,2,2,2,2,0,0,0,2,0,0,0,2,0,0,0,0,0,0,2,0,0,0,2\} \\
v_{22} & =c\{0,0,0,0,0,0,0,0,2,2,0,0,2,2,0,0,2,2,0,0,2,2,0,0\} \\
v_{23} & =c\{0,0,0,0,0,0,0,0,2,0,2,0,2,0,2,0,2,0,2,0,2,0,2,0\} \\
v_{24} & =c\{-3,1,1,1,1,1,1,1,1,1,1,1,1,1,1,1,1,1,1,1,1,1,1,1\}
\end{aligned}
$$

This is almost identical to the basis given in [68] except that we have taken a linear combination of the first two basis vectors of [68] so that all basis vectors have length squared four. One may easily check that the Gram matrix is an even unimodular matrix.

A generating set for the $\mathcal{C}$ sets of the Golay code can be read off from the above basis. For each of the basis vectors $v_{i}$ only one of the four sets $\mathcal{C}_{a}\left(v_{i}\right), a=1,2,3,4$ is nonempty. Let $S_{i}$ denote the corresponding $\mathcal{C}$-set. We will need:

$$
\begin{aligned}
S_{8} & =\{1,2,3,4,5,6,7,8\} \\
S_{20} & =\{1,2,5,7,9,12,21,24\} \\
S_{22} & =\{9,10,13,14,17,18,21,22\} \\
S_{23} & =\{9,11,13,15,17,19,21,23\}
\end{aligned}
$$


Two dodecads which are useful in our constructions are:

$$
\begin{aligned}
S_{20}+S_{22} & =\{1,2,5,7,10,12,13,14,17,18,22,24\} \\
S_{8}+S_{12}+S_{22} & =\{5,6,7,8,11,12,13,14,17,18,21,22\}
\end{aligned}
$$

We note that our choice of octads is slightly non-standard. See the table of octads in [66]. We also note that there are more efficient ways to generate them, as described in [18].

An important subgroup of $\mathrm{Co}_{0}$ for our considerations is the "monomial subgroup," isomorphic to $2^{12} \cdot M_{24}$. The action of this group is easily described in terms of the above model for the Leech lattice. $M_{24}$ is the group of permutations of coordinates preserving the extended binary Golay code. Moreover, one can flip coordinates on the $\mathcal{C}$-sets of the extended binary Golay code. Let $\epsilon_{S}$ be such a sign flip on a $\mathcal{C}$-set $S$. Then clearly

$$
\epsilon_{S_{1}} \epsilon_{S_{2}}=\epsilon_{S_{1}+S_{2}}
$$

and hence a set of generators for the subgroup of sign-flips is given by $\left\{\epsilon_{S_{a}}\right\}$ where $S_{a}$ runs over a basis for the Golay code. Therefore the group of sign-flips is isomorphic to $\mathbb{Z}_{2}^{12}$.

\section{Details On Lattice Computations: HM222}

\section{C.1 Construction Of $\Gamma^{22,6}$}

Recall from the discussion in 2.2 that construction of an even self-dual lattice of signature $(22,6)$ starts with a pair of isometric sublattices $\mathfrak{F}_{L} \subset \Gamma$ and $\mathfrak{F}_{R} \subset \Gamma_{8}$.

We first construct the lattice $\mathfrak{F}_{R}$ by choosing basis vectors

$$
\begin{aligned}
& \tilde{u}_{1}=u_{1}-u_{2}=(0,0,0,0,1,1,1,-1) \\
& \tilde{u}_{2}=u_{2}-u_{3}=\frac{1}{2}(-1,1,1,-3,-1,-1,-1,1)
\end{aligned}
$$

which are clearly in the span of the basis vectors for the Hadamard invariant sublattice $u_{1}, \cdots u_{4}$, have the desired Gram matrix

$$
Q^{222}=\left(\begin{array}{cc}
4 & -2 \\
-2 & 4
\end{array}\right) .
$$

and generate a primitive sublattice of $\Gamma_{8}$. We set $\mathfrak{F}_{R}=\mathbb{Z} \tilde{u}_{1}+\mathbb{Z} \tilde{u}_{2}$.

The dual lattice $\mathfrak{F}_{R}^{\vee}$ has a basis $\tilde{f}_{i}=G^{i j} \tilde{u}_{j}$ which gives

$$
\begin{aligned}
& \tilde{f}_{1}=\frac{1}{6}\left(2 \tilde{u}_{1}+\tilde{u}_{2}\right) \\
& \tilde{f}_{2}=\frac{1}{6}\left(\tilde{u}_{1}+2 \tilde{u}_{2}\right) .
\end{aligned}
$$

The generators of the discriminant group $\mathfrak{F}_{R}^{\vee} / \mathfrak{F}_{R}$ are as follows. Note that

$$
\begin{gathered}
\tilde{f}_{1}+\tilde{f}_{2}=\frac{1}{2}\left(\tilde{u}_{1}+\tilde{u}_{2}\right), \\
\tilde{f}_{1}-\tilde{f}_{2}=\frac{1}{6}\left(\tilde{u}_{1}-\tilde{u}_{2}\right) .
\end{gathered}
$$


Thus we can take the generators of $\mathfrak{F}_{R}^{\vee} / \mathfrak{F}_{R}=\mathbb{Z}_{2} \oplus \mathbb{Z}_{2} \oplus \mathbb{Z}_{3}$ to be

$$
\begin{aligned}
\tilde{c}_{1} & =\frac{1}{2}\left(\tilde{u}_{1}+\tilde{u}_{2}\right), \\
\tilde{c}_{2} & =\frac{1}{2} \tilde{u}_{1}, \\
\tilde{c}_{3} & =\frac{1}{3}\left(\tilde{u}_{1}-\tilde{u}_{2}\right) .
\end{aligned}
$$

Note that any $f^{*} \in \mathfrak{F}_{R}^{\vee}$ is expressible as an integer linear combination of the $\tilde{c}_{i}$ and $2 \tilde{c}_{1}, 2 \tilde{c}_{2}, 3 \tilde{c}_{3}$ are in $\mathfrak{F}_{R}$.

The lattice $\mathfrak{F}_{R}^{\perp}$ has basis vectors which may be chosen to be

$$
\begin{aligned}
\tilde{v}_{1} & =(1,0,1,0,0,0,0,0) \\
\tilde{v}_{2} & =(0,1,-1,0,0,0,0,0) \\
\tilde{v}_{3} & =(0,0,0,0,1,0,0,1) \\
\tilde{v}_{4} & =(0,0,0,0,0,-1,0,-1) \\
\tilde{v}_{5} & =\frac{1}{2}(1,-1,-1,-1,-1,1,-1,-1) \\
\tilde{v}_{6} & =\frac{1}{2}(-1,1,1,1,-1,1,-1,-1)
\end{aligned}
$$

with Gram matrix

$$
G_{\mathfrak{F}_{R}^{\perp}}^{222}=\left(\begin{array}{cccccc}
2 & -1 & 0 & 0 & 0 & 0 \\
-1 & 2 & 0 & 0 & 0 & 0 \\
0 & 0 & 2 & -1 & -1 & -1 \\
0 & 0 & -1 & 2 & 0 & 0 \\
0 & 0 & -1 & 0 & 2 & 0 \\
0 & 0 & -1 & 0 & 0 & 2
\end{array}\right)
$$

which we recognize as the Cartan matrix of $D_{4} \oplus A_{2}$. Thus $\mathfrak{F}_{R}^{\perp}=\Lambda_{\text {root }}\left(D_{4} \oplus A_{2}\right)$. The dual lattice $\left(\mathfrak{F}_{R}^{\perp}\right)^{\vee}$ has a basis $\tilde{g}_{i}=\left(G_{\mathfrak{F}_{R}^{\perp}}^{222}\right)^{i j} \tilde{u}_{j}$ which gives

$$
\begin{aligned}
\tilde{g}_{1} & =\frac{1}{3}(2,1,1,0,0,0,0,0) \\
\tilde{g}_{2} & =\frac{1}{3}(1,2,-1,0,0,0,0,0) \\
\tilde{g}_{3} & =(0,0,0,0,1,0,-1,0) \\
\tilde{g}_{4} & =\frac{1}{2}(0,0,0,0,1,-1,-1,-1) \\
\tilde{g}_{5} & =\frac{1}{4}(1,-1,-1,-1,1,1,-3,-1) \\
\tilde{g}_{6} & =\frac{1}{4}(-1,1,1,1,1,1,-3,-1) .
\end{aligned}
$$


It is not hard to see that one can choose the generators of the discriminant group $\left(\mathfrak{F}_{R}^{\perp}\right)^{\vee} / \mathfrak{F}_{R}^{\perp} \simeq$ $\mathbb{Z}_{2} \oplus \mathbb{Z}_{2} \oplus \mathbb{Z}_{3}$ to be

$$
\begin{aligned}
& \tilde{d}_{1}=\tilde{v}_{5}, \\
& \tilde{d}_{2}=\tilde{v}_{6}, \\
& \tilde{d}_{3}=\tilde{v}_{1} .
\end{aligned}
$$

Since $\left(c_{1}^{2}, c_{2}^{2}, c_{3}^{2}\right)=(1,1,4 / 3)$ and $\left(d_{1}^{2}, d_{2}^{2}, d_{3}^{2}\right)=(1,1,2 / 3)$ we have $c_{i}^{2}=-d_{i}^{2} \bmod 2$ for $i=1,2,3$ so the map $\left(c_{1}, c_{2}, c_{3}\right) \rightarrow\left(d_{1}, d_{2}, d_{3}\right)$ gives an isometry $\mathcal{D}_{-}\left(\mathfrak{F}_{R}\right) \cong \mathcal{D}_{+}\left(\mathfrak{F}_{R}^{\perp}\right)$ as described in subsection 2.2 .

The construction of $\mathfrak{F}_{L}$ and $\left(\mathfrak{F}_{L}^{\perp}\right)^{\vee}$, the lattice $\Gamma$ and the invariant lattice $\Gamma^{g}$ as well as the count of massless states in the twisted sector for HM22 follows very similar lines to the computations for HM224 for which details are provided in the following section. As a result we omit them here and will provide them upon request.

\section{Details On Lattice Computations: HM224}

\section{D.1 Construction Of $\Gamma^{22,6}$}

Let us begin with the construction of $\mathfrak{F}_{R}$ and $\mathfrak{F}_{R}^{\perp}$. We take $\mathfrak{F}_{R}$ to be generated by

$$
\begin{aligned}
& \tilde{u}_{1}=(1,0,0,1,1,0,0,1) \\
& \tilde{u}_{2}=(1,1,1,-1,0,0,0,0)
\end{aligned}
$$

These are fixed by the Hadamard involution, generate the required Gram matrix, and generate a primitive sublattice.

It is straightforward to compute a basis for the orthogonal lattice $\mathfrak{F}_{R}^{\perp}$ :

$$
\begin{aligned}
& \tilde{v}_{1}=\{0,0,0,0,0,1,1,0\} \\
& \tilde{v}_{2}=(1 / 2)\{1,-1,-1,-1,1,-1,-1,-1\} \\
& \tilde{v}_{3}=\{0,0,0,0,-1,0,0,1\} \\
& \tilde{v}_{4}=\{0,1,-1,0,0,0,0,0,\} \\
& \tilde{v}_{5}=(1 / 2)\{1,-1,1,1,-1,1,-1,-1\} \\
& \tilde{v}_{6}=\{0,0,0,0,0,-1,1,0\}
\end{aligned}
$$

The Gram matrix of the $\tilde{v}_{i}$ is

$$
\left(\begin{array}{cccccc}
2 & -1 & 0 & 0 & 0 & 0 \\
-1 & 2 & -1 & 0 & 0 & 0 \\
0 & -1 & 2 & 0 & 0 & 0 \\
0 & 0 & 0 & 2 & -1 & 0 \\
0 & 0 & 0 & -1 & 2 & -1 \\
0 & 0 & 0 & 0 & -1 & 2
\end{array}\right)
$$

which we recognize as the Cartan matrix for $A_{3} \oplus A_{3}$. This makes it clear that the discriminant group $\mathcal{D}\left(\mathfrak{F}_{R}^{\perp}\right)$ is $\mathbb{Z}_{4} \oplus \mathbb{Z}_{4}$. 
By inverting the Gram matrix of the $\tilde{u}_{i}$ we can obtain explicit generators of $\mathcal{D}\left(\mathfrak{F}_{R}\right)$

$$
\begin{aligned}
& \tilde{c}_{1}=(1 / 4)\{1,0,0,1,1,0,0,1\} \\
& \tilde{c}_{2}=(1 / 4)\{1,1,1,-1,0,0,0,0\}
\end{aligned}
$$

and similarly we find explicit generators of $\mathcal{D}\left(\mathfrak{F}_{R}^{\perp}\right)$ :

$$
\begin{aligned}
\tilde{d}_{1} & =(1 / 4)\{1,-1,-1,-1,0,2,2,0\} \\
\tilde{d}_{2} & =(1 / 4)\{1,2,-2,1,-1,0,0,-1\} .
\end{aligned}
$$

Since $\tilde{c}_{1}^{2}=\tilde{c}_{2}^{2}=1 / 4$ while $\tilde{d}_{1}^{2}=\tilde{d}_{2}^{2}=3 / 4$ and $\tilde{d}_{1} \cdot \tilde{d}_{2}=0$ we can construct the isometry of discriminant groups $\psi_{R}$ by mapping $\left(\tilde{c}_{1}, \tilde{c}_{2}\right) \rightarrow\left(\tilde{d}_{1}+2 \tilde{d}_{2}, 2 \tilde{d}_{1}+\tilde{d}_{2}\right)$.

On the left-moving side we need to proceed a little more indirectly to avoid having to compute a basis for the 22 -dimensional sublattice $\mathfrak{F}_{L}^{\perp} \subset \Lambda$. Now $\mathfrak{F}_{L}$ is spanned by $v_{1}, v_{2}$. Therefore $\mathcal{D}_{+}\left(\mathfrak{F}_{L}\right)$ is just $\mathbb{Z} / 4 \mathbb{Z} \oplus \mathbb{Z} / 4 \mathbb{Z}$ with $q\left(n_{1}, n_{2}\right)=\frac{n_{1}^{2}}{4}+\frac{n_{2}^{2}}{4} \bmod 2 \mathbb{Z}$. Define

$$
\begin{array}{ll}
k_{i}:=v_{1} \cdot v_{i} & 1 \leq i \leq 24 \\
\ell_{i}:=v_{2} \cdot v_{i} & 1 \leq i \leq 24
\end{array}
$$

Now define vectors in $\mathfrak{F}_{L}^{\perp} \otimes \mathbb{Q}$ :

$$
\rho_{a}:=-\frac{k_{a}}{4} v_{1}-\frac{\ell_{a}}{4} v_{2}+v_{a} \quad 3 \leq a \leq 24
$$

One can check that these span $\left(\mathfrak{F}_{L}^{\perp}\right)^{\vee}$. Moreover the isomorphism

$$
\psi_{L}: \mathcal{D}_{-}\left(\mathfrak{F}_{L}^{\perp}\right) \rightarrow \mathcal{D}_{+}\left(\mathfrak{F}_{L}\right) \cong \mathbb{Z}_{4} \oplus \mathbb{Z}_{4}
$$

must take the form

$$
\rho_{a} \rightarrow\left(k_{a} \bmod 4, \ell_{a} \bmod 4\right)
$$

Moreover

$$
\rho_{a} \cdot \rho_{b}=-\frac{k_{a} k_{b}}{4}-\frac{\ell_{a} \ell_{b}}{4}+v_{a} \cdot v_{b}
$$

Therefore, if we use the quadratic function

$$
q(\bar{v}):=-v^{2} \bmod 2
$$

we get an isometry of discriminant groups since we can form the integral linear combinations

$$
\gamma_{a}:=\rho_{a}+\rho_{24} \quad a \in \Sigma_{1}:=\{3,4,5,6,7,9,10,11,13,17\}
$$

with $(k, \ell)=(0,1)$ and

$$
\gamma_{b a}:=\rho_{b}-\left(\rho_{a}+\rho_{24}\right) \quad a \in \Sigma_{1} \quad \& \quad b \in \Sigma_{2}:=\{15,16,18,19\}
$$

with $(k, \ell)=(1,0)$. We can now take any of the above $\gamma_{a}$ for the glue vector $d_{1}$ and any of $\gamma_{b a}$ for the glue vector $d_{2}$.

Thus, finally, we have explicitly constructed the CSS compactification:

$$
\Gamma^{22,6}:=\amalg_{r=0,1,2,3} \amalg_{r^{\prime}=0,1,2,3}\left(\Gamma_{0}^{22,6}+r\left(d_{1} ; \tilde{d}_{1}\right)+r^{\prime}\left(d_{2} ; \tilde{d}_{2}\right)\right)
$$

where $\Gamma_{0}^{22,6}=\left(\mathfrak{F}_{L}^{\perp} ; 0\right) \oplus\left(0 ; \mathfrak{F}_{R}^{\perp}\right)$. 


\section{D.2 Doomed To Fail?}

One can check that $g=\left(g_{L}^{A, B, C} ; \sigma_{2}\right)$ acts as the identity matrix modulo $\Gamma_{0}^{22,6}$ on all the glue vectors. Therefore $g$ is an involutive automorphism of the lattice $\Gamma^{22,6}$. Moreover, one can check for $g_{L}^{A}$ and $g_{L}^{B}$ there are vectors with $(p, g \cdot p)$ odd, while for $g_{L}^{C}$ all inner products $(p, g \cdot p)$ are even. (Checking the above statements involves some nontrivial computation - details are available upon request.) Based on the analysis in 41] we conclude that the lattice involutions for models A,B lift to symmetries of order four of the lattice CFT while for model $\mathrm{C}$ the symmetry of the CFT remains order two.

\section{D.3 The Invariant Lattice $\Gamma^{g}$ And Its Discriminant Group}

We briefly describe the computation of $\Gamma^{g}$ for these models following the technique discussed in section 3.7. For model B we construct the lattice $\left(1+g_{B}\right) \Gamma$ and then check that (3.35) has no non-trivial solutions which implies that $\Gamma^{g_{B}}=\left(1+g_{B}\right) \Gamma$. A set of basis vectors for $\Gamma^{g_{B}}$ consists of the vectors $\left(w_{a}, ; \tilde{w}_{a}\right), a=1,, 8$ with

$$
\begin{aligned}
& w_{1}=c\{0,0,0,0,0,0,0,0,0,0,0,0,0,0,0,0,0,0,0,0,0,0,0,0\} \\
& w_{2}=c\{0,0,0,0,0,0,0,0,0,0,0,0,0,0,0,0,0,0,0,0,0,0,0,0\} \\
& w_{3}=c\{0,0,0,4,0,0,0,0,0,0,0,0,0,0,0,0,0,0,0,0,0,0,0,0\} \\
& w_{4}=c\{0,0,2,2,2,2,2,2,0,0,0,0,0,0,0,0,0,0,0,0,0,0,0,0\} \\
& w_{5}=c\{0,0,0,0,4,0,0,0,0,0,0,0,0,0,0,0,0,0,0,0,0,0,0,0\} \\
& w_{6}=c\{0,0,0,0,0,4,0,0,0,0,0,0,0,0,0,0,0,0,0,0,0,0,0,0\} \\
& w_{7}=c\{0,0,0,0,0,0,4,0,0,0,0,0,0,0,0,0,0,0,0,0,0,0,0,0\} \\
& w_{8}=c\{0,0,0,0,0,0,0,4,0,0,0,0,0,0,0,0,0,0,0,0,0,0,0,0\} \\
& \tilde{w}_{1}=\{0,0,0,0,1,1,1,-1\} \\
& \tilde{w}_{2}=\{1,0,0,1,-1,0,0,-1\} \\
& \tilde{w}_{3}=\{1 / 2,0,0,1 / 2,0,1 / 2,1 / 2,-1\} \\
& \tilde{w}_{4}=\{0,0,0,0,1 / 2,1 / 2,1 / 2,-1 / 2\} \\
& \tilde{w}_{5}=\{1 / 2,0,0,1 / 2,0,1 / 2,1 / 2,-1\} \\
& \tilde{w}_{6}=\{1 / 2,0,0,1 / 2,0,1 / 2,1 / 2,-1\} \\
& \tilde{w}_{7}=\{1 / 2,0,0,1 / 2,0,1 / 2,1 / 2,-1\} \\
& \tilde{w}_{8}=\{1 / 2,0,0,1 / 2,0,1 / 2,1 / 2,-1\}
\end{aligned}
$$

For model $A$ on the other hand there are 63 non-trivial solutions to (3.35) and to construct $\Gamma^{g_{A}}$ we produce an overcomplete basis by adjoining the solutions to (3.35) to the basis vectors of $\left(1+g_{A}\right) \Gamma$ and then compute a basis from this over complete set. We then 
find for the Gram matrix of $\Gamma^{g_{A}}$

$$
\operatorname{Gram}\left(\Gamma^{g_{A}}\right)=\left(\begin{array}{cccccccccccccccc}
-4 & 0 & -2 & -2 & -2 & -2 & -2 & -2 & 2 & -2 & 2 & 2 & 2 & -2 & -2 & 0 \\
0 & -4 & -2 & 0 & 0 & 0 & -2 & -2 & 0 & -2 & 0 & 0 & 0 & -2 & -2 & 2 \\
-2 & -2 & 0 & -1 & 0 & -1 & -2 & -2 & 0 & -2 & 0 & 0 & 2 & -2 & -2 & 2 \\
-2 & 0 & -1 & 2 & 0 & 0 & -1 & 0 & 2 & 0 & 2 & 2 & 0 & -1 & 0 & -1 \\
-2 & 0 & 0 & 0 & 2 & 0 & -1 & -1 & 0 & 0 & 0 & 0 & 2 & 0 & -1 & 1 \\
-2 & 0 & -1 & 0 & 0 & 2 & 0 & -1 & 2 & -1 & 0 & 0 & 0 & 0 & 0 & -1 \\
-2 & -2 & -2 & -1 & -1 & 0 & 0 & -2 & 1 & -2 & 1 & 0 & 0 & -2 & -2 & 1 \\
-2 & -2 & -2 & 0 & -1 & -1 & -2 & 0 & 1 & -2 & 1 & 2 & 0 & -2 & -2 & 1 \\
2 & 0 & 0 & 2 & 0 & 2 & 1 & 1 & 2 & 1 & 0 & 0 & -2 & 1 & 2 & -2 \\
-2 & -2 & -2 & 0 & 0 & -1 & -2 & -2 & 1 & 0 & 2 & 1 & 1 & -2 & -2 & 1 \\
2 & 0 & 0 & 2 & 0 & 0 & 1 & 1 & 0 & 2 & 2 & 0 & -2 & 0 & 1 & -1 \\
2 & 0 & 0 & 2 & 0 & 0 & 0 & 2 & 0 & 1 & 0 & 2 & -2 & 1 & 1 & -1 \\
2 & 0 & 2 & 0 & 2 & 0 & 0 & 0 & -2 & 1 & -2 & -2 & 2 & 1 & 1 & 1 \\
-2 & -2 & -2 & -1 & 0 & 0 & -2 & -2 & 1 & -2 & 0 & 1 & 1 & 0 & -2 & 1 \\
-2 & -2 & -2 & 0 & -1 & 0 & -2 & -2 & 2 & -2 & 1 & 1 & 1 & -2 & 0 & 0 \\
0 & 2 & 2 & -1 & 1 & -1 & 1 & 1 & -2 & 1 & -1 & -1 & 1 & 1 & 0 & 2
\end{array}\right)
$$

with determinant $2^{10}$.

\section{D.4 The Massless Charged Twisted Sector Ground States}

In model A there are massless charged states appearing in the twisted sector of the orbifold. To count these states we need to compute the theta function of the dual of the invariant lattice, $\left(\Gamma^{g_{A}}\right)^{\vee}$. In particular we want to count states with $p_{R}=0$ and $p_{L}^{2}=1$. To do this we first project onto the sublattice of states with $p_{R}=0$ and compute its Gram matrix. This leads to

$$
\left(\begin{array}{cccccccccccccc}
1 & -\frac{1}{2} & 0 & \frac{1}{2} & 0 & 0 & 0 & 0 & \frac{1}{2} & \frac{1}{2} & 0 & 0 & 0 & 0 \\
-\frac{1}{2} & 2 & -1 & -1 & 0 & -\frac{1}{2} & 0 & 0 & -1 & -1 & 0 & \frac{1}{2} & -\frac{1}{2} & 0 \\
0 & -1 & 2 & -1 & 0 & 0 & \frac{1}{2} & -\frac{1}{2} & 0 & 0 & -1 & -\frac{1}{2} & \frac{1}{2} & -\frac{1}{2} \\
\frac{1}{2} & -1 & -1 & 4 & 0 & \frac{1}{2} & -1 & 0 & 2 & 2 & 2 & -\frac{1}{2} & -\frac{1}{2} & 1 \\
0 & 0 & 0 & 0 & 1 & 0 & 0 & 0 & 0 & \frac{1}{2} & \frac{1}{2} & 0 & 0 & 0 \\
0 & -\frac{1}{2} & 0 & \frac{1}{2} & 0 & 1 & 0 & 0 & \frac{1}{2} & 0 & \frac{1}{2} & 0 & 0 & 0 \\
0 & 0 & \frac{1}{2} & -1 & 0 & 0 & 1 & 0 & -\frac{1}{2} & -\frac{1}{2} & -\frac{1}{2} & 0 & 0 & 0 \\
0 & 0 & -\frac{1}{2} & 0 & 0 & 0 & 0 & 1 & -\frac{1}{2} & 0 & 0 & 0 & 0 & 0 \\
\frac{1}{2} & -1 & 0 & 2 & 0 & \frac{1}{2} & -\frac{1}{2} & -\frac{1}{2} & 2 & 1 & 1 & 0 & 0 & \frac{1}{2} \\
\frac{1}{2} & -1 & 0 & 2 & \frac{1}{2} & 0 & -\frac{1}{2} & 0 & 1 & 2 & 1 & -\frac{1}{2} & 0 & \frac{1}{2} \\
0 & 0 & -1 & 2 & \frac{1}{2} & \frac{1}{2} & -\frac{1}{2} & 0 & 1 & 1 & 2 & 0 & -\frac{1}{2} & \frac{1}{2} \\
0 & \frac{1}{2} & -\frac{1}{2} & -\frac{1}{2} & 0 & 0 & 0 & 0 & 0 & -\frac{1}{2} & 0 & 1 & 0 & 0 \\
0 & -\frac{1}{2} & \frac{1}{2} & -\frac{1}{2} & 0 & 0 & 0 & 0 & 0 & 0 & -\frac{1}{2} & 0 & 1 & 0 \\
0 & 0 & -\frac{1}{2} & 1 & 0 & 0 & 0 & 0 & \frac{1}{2} & \frac{1}{2} & \frac{1}{2} & 0 & 0 & 1
\end{array}\right)
$$

The theta function of the above Gram matrix is

$$
1+28 q^{1 / 2}+2156 q+\cdots
$$


which leads to the claim in Table 2 that there are 28 massless states appearing in the twisted sector for HM224 model A. Inspection (using Magma) of the actual short vectors (with $p_{L}^{2}=1$ ) shows that there are 14 independent short vectors, hence $\mathcal{L}_{1,0}=14$.

\section{E. A Case Of Mistaken Moonshine}

The space of DH states $\mathcal{V}^{\text {BPS }}$ in a toroidal heterotic string compactification is graded by the Narain lattice and consists of right-moving ground states and any left-moving state satisfying level-matching [19]. Using light cone quantization the space of physical BPS states as a vector space is

$$
\mathcal{V}^{\mathrm{BPS}}=\oplus_{P \in \Gamma} \mathcal{D}_{P}^{\mathrm{BPS}} \otimes \tilde{\mathcal{R}}
$$

where $\tilde{\mathcal{R}}$ are the right-moving ground states of the 10-dimensional superstring. They transform as $8_{v} \oplus 8_{s}$ under the Spin(8) automorphism of the transverse space to the lightcone. We refer to the spaces $\mathcal{D}_{P}^{\mathrm{BPS}}$ as the $B P S$ degeneracy spaces. In a light-cone gauge formalism, the BPS degeneracy spaces $\mathcal{D}_{P}^{\mathrm{BPS}}$ can be identified with the level $N=N_{P}$ subspace of a Fock space of 24 chiral bosons with

$$
N_{P}:=1+\frac{1}{2}\left(P_{R}^{2}-P_{L}^{2}\right)
$$

Remark: For $d=4$ heterotic/type II duality states that the DH states can be identified with the boundstates of D4-D2-D0 branes for the type IIA string compactified on $\mathbb{M}^{1,5} \times K 3$. These are precisely the BPS states that were investigated in [48], especially with regard to their $\mathfrak{s o}(4) \cong \mathfrak{s u}(2) \oplus \mathfrak{s u}(2)$ quantum numbers. Physically, those are the quantum numbers under a subgroup of the little group of a corresponding unitary representation of the Lorentz group $\operatorname{Spin}_{0}(1,5)$. Thus, it is possible to read the comments in this section, for $d=4$, as comments about the $\mathfrak{s o}(4)$ representation content of these D-brane boundstates.

In what follows we will consider several Fock spaces generated by chiral bosons valued in a $G$-module $V$ for various groups $G$. Thus we denote

$$
\mathcal{F}_{q} V:=\operatorname{Sym}^{\bullet}\left(q V \oplus q^{2} V \oplus q^{3} V \oplus \cdots\right)=\operatorname{Sym}_{q}^{\bullet}(V) \otimes \operatorname{Sym}_{q^{2}}^{\bullet}(V) \otimes \operatorname{Sym}_{q^{3}}^{\bullet}(V) \otimes \cdots
$$

where $q$ keeps track of the level $N$, and

$$
\operatorname{Sym}_{t}^{\bullet}(V):=\oplus_{j=0}^{\infty} t^{j} \operatorname{Sym}^{j}(V)
$$

Note that if we consider $\mathcal{F}_{q}(V)$ as a $q$-expansion with coefficients in the monoid of $G$ modules then we can define an inverse in the space of $q$-expansions with coefficients in the representation ring of $G$. This will be a key idea in the discussion below. 


\section{E.1 What Is Moonshine?}

The dimensions of the BPS degeneracy spaces $\mathcal{D}_{P}^{\mathrm{BPS}}$ are naturally written as sums of dimensions of irreducible representations of $O(24)$. When we consider the compactification (2.1) an $O(d)$ subgroup is selected as a subgroup of the little group of a particle and hence the subgroup $O(24-d) \times O(d) \subset O(24)$ is distinguished. Of course, the continuous symmetry is broken by the Narain lattice, but if one only studies BPS degeneracies and not, for example, the algebra of BPS states (in the heterotic theory) or auto-equivalences of the derived category (in the type IIA theory) then that breaking is not visible. All the crystal symmetry groups $G_{L}$ in the CSS compactifications are subgroups of $O(24-d)$, so it would be silly to speak of "Moonshine" with respect to these groups. Nevertheless, one can ask if there are discrete groups not in $O(24-d)$ that act on the spaces of BPS states in a way compatible with modularity. Moonshine is concerned with the latter phenomenon.

We will presently be more precise about the last sentence of the previous paragraph, but first a specific example will help clarify what we are speaking about. Consider the case of $d=1$. From Table 1 of 43 we see that the largest CSS symmetry for $d=1$ is $\mathrm{Co}_{2}$, the subgroup of $\mathrm{Co}_{0}$ that stabilizes a Leech vector of length-squared equal to four. The space of BPS states, $\mathcal{V}^{\mathrm{BPS}}$ as a representation of $\mathrm{Co}_{2} \times O(1)$, can be obtained from the Fock space

$$
\mathcal{F}_{q}\left(V_{23} \otimes \mathbf{T} \oplus \mathbf{1} \otimes \mathbf{S}\right)
$$

where $V_{23}$ is the 23-dimensional representation of $\mathrm{Co}_{2}$ and $\mathbf{T}$ and $\mathbf{S}$ are the trivial and sign representations of $O(1)$. The $q$-expansion is

$$
\begin{gathered}
\mathcal{F}\left(V_{23} \otimes \mathbf{T} \oplus \mathbf{1} \otimes \mathbf{S}\right) 1 \oplus q\left[V_{23} \otimes \mathbf{T} \oplus \mathbf{1} \otimes \mathbf{S}\right] \\
\oplus q^{2}\left\{\left[\operatorname{Sym}^{2}\left(V_{23}\right) \oplus V_{23} \oplus \mathbf{1}\right] \otimes \mathbf{T} \oplus\left[V_{23} \oplus \mathbf{1}\right] \otimes \mathbf{S}\right\} \\
\oplus q^{3}\left\{\left[\operatorname{Sym}^{3}\left(V_{23}\right) \oplus V_{23}^{\otimes 2} \oplus 2 \cdot V_{23} \oplus \mathbf{1}\right] \otimes \mathbf{T} \oplus\left[\operatorname{Sym}^{2}\left(V_{23}\right) \oplus 2 \cdot V_{23} \oplus 2 \mathbf{1}\right] \otimes \mathbf{S}\right\} \\
\oplus \cdots
\end{gathered}
$$

Indeed $\mathrm{Co}_{2} \times O(1)$ will be an automorphism of the algebra of BPS states. However, if we just study the BPS degeneracies $\mathcal{D}_{P}^{\mathrm{BPS}}$ as representations of $\mathrm{Co}_{2} \times O(1)$ we might as well study them as representations of $O(23) \times O(1)$ since we can simply regard $V_{23}$ as the vector representation of $O(23)$ : If we just look at the degeneracies there is nothing special about the discrete group $\mathrm{Co}_{2} \subset O(23)$.

However, we can ask if there is a non-manifest action of the larger group $\mathrm{Co}_{0}$ on the BPS degeneracy spaces $\mathcal{D}_{P}^{\mathrm{BPS}}$. Note that $\mathrm{Co}_{0}$ does not have a nontrivial 23-dimensional representation - so there is no manifest $\mathrm{Co}_{0} \times O(1)$ action on (E.6) (compatible with the pullback to $\mathrm{Co}_{2} \times O(1)$ ). One way to investigate this is to play the "SumDimension game" that goes back to McKay's observations about the Monster and which was used to such great effect in [27]. The rules of the game in our context (for $d=1$ ) are: 
SumDim1: List the dimensions of the irreducible representations of $\mathrm{Co}_{0}$ :

$$
\operatorname{Irrep}\left(\mathrm{Co}_{0}\right)=\{\mathbf{1}, \mathbf{2 4}, \mathbf{2 7 6}, \mathbf{2 9 9}, \mathbf{1 7 7 1}, \mathbf{2 0 2 4}, \mathbf{2 5 7 6}, \mathbf{4 5 7 6}, \ldots\}
$$

SumDim2: Decompose the coefficients of $q^{n} \mathbf{T}$ and $q^{n} \mathbf{S}$ into the simplest possible nonnegative combinations of the integers (E.7). "Simplest" means "using the fewest number of parts." Such minimal decompositions are in general not unique. Choose one.

SumDim3: Use "simple" virtual representations for "small" degeneracies. These are typically associated with massless or exceptional representations. In our $d=1$ example the "small" representations are the 23-dimensional representations in the coefficient of $q \mathbf{T}$ and the coefficient of $q^{2} \mathbf{S}$.

As an illustration of rule two consider the coefficient of $\mathbf{T}$ at level 2:

$$
\operatorname{dim}\left(\operatorname{Sym}^{2}\left(V_{23}\right) \oplus V_{23} \oplus \mathbf{1}\right)=300
$$

Referring to (E.7) we can decompose

$$
300=299+1
$$

or, equally well,

$$
300=276+24
$$

Either choice allows us to define a $\mathrm{Co}_{0}$ action on that component of the space of BPS states. Once we have made such a choice for all the massive levels we have defined the Fock space as a $\mathrm{Co}_{0} \times O(1)$-module. Of course, there are infinitely many such choices and we must search for some principle that tells us which, if any, are interesting.

The criterion we will use is modularity of the $\mathrm{Co}_{0} \times O(1)$ characters: We will say that the space of DH states exhibits "Moonshine for a group $G$ " if that space of states has a G-module structure with $G$ commuting with the level operator and such that the graded character of $g \in G$ is a modular form for $\Gamma_{0}(m)$ where $g$ has order $m$.

\section{E.2 Virtual Representations}

It is easy to make Fock spaces of virtual representations whose characters are modular. For example, returning to the example of $d=1$ discussed above, if we replace

$$
V_{23} \rightarrow V_{24}-\mathbf{1}
$$

and interpret $V_{24}$ as the 24-dimensional representation of $\mathrm{Co}_{0}$ then the Fock space

$$
\mathcal{F}\left(\left(V_{24}-\mathbf{1}\right) \otimes \mathbf{T} \oplus \mathbf{1} \otimes \mathbf{S}\right)=\mathcal{F}\left(V_{24} \otimes \mathbf{T}\right) \otimes \frac{\mathcal{F}(\mathbf{1} \otimes \mathbf{S})}{\mathcal{F}(\mathbf{1} \otimes \mathbf{T})}
$$

regarded as a $q$-expansion with coefficients in the representation ring of $\mathrm{Co}_{0} \times O(1)$ makes perfect sense. Moreover, its graded characters

$$
\operatorname{Tr} g q^{L_{0}-1}
$$


will be modular forms for $\Gamma_{0}(m)$ if $g$ has order $m$.

The expression (E.12) is, a priori, only a $q$-expansion of virtual representations. However, it is possible to show that in fact, with the exception of the coefficient of $\mathbf{T}$ at level 1 and $\mathbf{S}$ at level 2 all the massive representations are in fact positive combinations of $\mathrm{Co}_{0}$ irreps! But, dear reader, we hasten to add that this is a somewhat silly form of Moonshine because we can also regard $V_{24}$ as the vector representation of $O(24)$ and the same positivity holds for (E.12) as a representation of $O(24) \times O(1)$ : Again, there is nothing magical about the discrete group $\mathrm{Co}_{0} \times O(1) \subset O(24) \times O(1)$.

There are two ways to prove this claim of positivity. One proof identifies the extension of $O(23) \times O(1)$ symmetry to $O(24) \times O(1)$ symmetry as the familiar fact that in lightcone gauge only the massless little group acts linearly on the transverse oscillators and the extension to the generators of the massive little group involve expressions that are nonlinear in oscillators. In more detail, the argument goes as follows:

Consider $(D-1)$ bosons $\alpha^{i}, i=1, \ldots, D-1$ and one extra boson $\beta$. We consider the Fock space $\mathcal{F}(V)$ based on the $D$-dimensional representation of $O(D-1) \times O(1)$ :

$$
V=(\mathbf{D}-\mathbf{1}) \otimes \mathbf{T} \oplus \mathbf{1} \otimes \mathbf{S}
$$

where $\mathbf{T}$ is the trivial representation of $O(1)$ and $\mathbf{S}$ is the sign representation of $O(1)$.

Since $V$ is $D$-dimensional, if we forget about the $O(1)$ quantum number of $\beta$ then the level $N$ subspace of $\mathcal{F}(V)$ is clearly a representation of $O(D)$, and under the natural inclusion of $O(D-1) \hookrightarrow O(D)$ it becomes the same $O(D-1)$ representation as the pullback under $O(D-1) \hookrightarrow O(D-1) \times O(1)$. A nontrivial fact - which is clear by thinking of the space $V$ as a transverse space in a light-cone gauge formulation of string theory in $\mathbb{M}^{1, D+1}$ (for $D \leq 24$ ) is that for $N>1, \mathcal{F}^{N}(V)$ is in fact a representation of $O(D+1)$ such that, under the inclusion of $O(D) \hookrightarrow O(D+1)$ it pulls back to the natural $O(D)$ representation noted above.

Thus, unlike the $O(D)$ action, there is an $O(D+1)$ action not induced from a linear action on $V$. We can now define an action by an $O(1)$ subgroup of $O(D+1)$ by counting the number of $\beta$ oscillators modulo two. Of course $O(1) \cong \mathbb{Z}_{2}$, viewed multiplicatively and we consider the subgroup defined by identifying the nontrivial generator as

$$
\sigma=(-1)^{N_{\beta}}
$$

The centralizer of this involution in $O(D+1)$ is a copy of $O(D)$, and therefore $\mathcal{F}^{N}(V)$ is a true representation of $O(D) \times O(1)$.

A second, more computational argument shows that in fact the positivity persists for all $D$, not just $D \leq 24$.

\section{E.3 $d=4$ : Mistaken Moonshine}

Let us now return to the the case of $d=4$, corresponding, via heterotic/type II duality to the degeneracies studied in [48]. We are guaranteed $O(20) \times O(4)$ symmetry of BPS

degeneracy spaces $\mathcal{D}_{P}^{\mathrm{BPS}}$. Let us ask if these spaces are, in any natural way, in fact representations of $M_{24} \times O(4)$. This would be nontrivial since the smallest nontrivial representation of $M_{24}$ is 23-dimensional. 
Given the surprising cancellations observed in the $d=1$ case the first thing we might ask is whether the analogous Fock space of virtual representations exhibits similar cancellations. Since $M_{24}$ has a 23-dimensional representation $V_{23}$ we consider

$$
\left.\mathcal{F}_{q}\left((\mathbf{2 3}-3 \mathbf{1}) \otimes \mathbf{1} \oplus \mathbf{1} \otimes V_{4}\right)\right)=\mathcal{F}_{q}(\mathbf{2 3} \otimes \mathbf{1}) \frac{\mathcal{F}_{q}\left(\mathbf{1} \otimes V_{4}\right)}{\mathcal{F}_{q}(\mathbf{1} \otimes \mathbf{1})^{3}}
$$

where $V_{4}$ is the vector representation of $O(4)$. Once again, the virtue of this construction is that the character of elements of $M_{24} \times O(4)$ of finite order $m$ are guaranteed to be modular forms for $\Gamma_{0}(m)$. In this sense, equation (E.16) exhibits "Moonshine" for $M_{24} \times O(4)$.

It is straightforward to expand $(\mathbb{E . 1 6})$ in $q$. Let us for example consider the level 2 degeneracy of the singlet of $O(4)$. This degeneracy is 231, which happens to be the dimension of an irrep of $M_{24}$. Unfortunately, the explicit virtual representation of $M_{24}$ in (E.16) turns out to be

$$
S^{2} V-2 V+1=V_{252}-V_{23}+2
$$

It indeed has net dimension 231, but it is not a true representation.

One might still ask if, nevertheless, one of the solutions of the SumDimension game nevertheless turns out to be modular. We believe that the answer to this question is also negative. To show this we begin by playing the SumDimension game at low levels. The dimensions of irreducible representations of $M_{24}$ are

$$
\begin{aligned}
\operatorname{Irrep}\left(M_{24}\right)= & \{1,23,45,45,231,231,252,253,483,770,770,990,990, \\
& 1035,1035,1035,1265,1771,2024,2277,3312,3520,5313,5544,5796,10395\}
\end{aligned}
$$

Next we study the possible characters of involutions in $M_{24}$ at low level. Because a given degeneracy can have several possible "simplest" decompositions as sums of dimensions of irreps of $M_{24}$ already at level four there are twelve possible characters for the conjugacy classes of involutions of $M_{24}$ commonly denoted $2 A$ and $2 B$. One finds, for example

$$
Z_{2 A}=1 / q+8+36 q+144 q^{2}+282 q^{3}+\cdots
$$

and eleven other possibilities, all differing in the coefficient of $q^{3}$. A similar story holds for $Z_{2 B}$. Now, just based on this meager information it might seem impossible to rule out modularity for $\Gamma_{0}(2)$. After all, we do not know the weight, the multiplier system, nor the higher order terms in the $q$-expansion!

The trick to showing that $Z_{2 A}$ and its cousins cannot be modular is based on studying the behavior of the character in the vicinity of $\tau_{0}:=(1+\mathrm{i}) / 2$. Recall that $\Gamma_{0}(2)$ is generated by $T$ and $S T^{2} S$, the latter transformation acting as

$$
\tau^{\prime}=S T^{2} S \cdot \tau=\frac{\tau}{1-2 \tau}
$$

In particular, note that $S T^{2} S \cdot \tau_{0}=\tau_{0}-1$. Since (E.19) is an expansion in integer powers of $q$ it is invariant under $T$. Thus, $\tau_{0}$ is effectively a fixed point of $S T^{2} S$ and one can 
determine the multiplier system from the weight, as explained in Appendix $\mathrm{F}$. Moreover, if $\tau=\tau_{0}+\delta \tau$ with $\delta \tau$ small then

$$
\tau^{\prime}=\tau_{0}-1-\delta \tau+\mathcal{O}\left(\delta \tau^{2}\right)
$$

and so expanding the transformation formula in powers of $\delta \tau$ we find that the weight can be deduced from

$$
w=\lim _{\epsilon \rightarrow 0} \frac{1}{2 \epsilon} \log \left|\frac{Z\left(\tau_{0}-\mathrm{i} \epsilon\right)}{Z\left(\tau_{0}+\mathrm{i} \epsilon\right)}\right|
$$

Finally, for $\tau=\tau_{0}$ the numerical value of $q$ is $q=\exp [2 \pi \mathbf{i} \tau]=-e^{-\pi}=-0.0432139 \ldots$ Since the generating function of the dimensions is rapidly convergent for this value of $q$ we have every reason to expect that the graded characters are also rapidly convergent and therefore we can compute $w$ to a good approximation just from the first four terms given in (E.19) and its cousins. The result of this computation is a non-half-integral weight $w=-8.4 \ldots$ in all cases the series is converging to a complicated decimal expansion. (The value of $w$ for the twelve cases all differ only in the third significant figure.) Therefore, if $Z_{2 A}$ is to be modular then it cannot have a half-integral weight. ${ }^{10}$ While it is possible to develop a theory of automorphic forms for $S L(2, \mathbb{Z})$ of general complex weight, all Moonshine and conformal field theory constructions of which we are aware involve forms of half-integral weight. It thus seems extremely unlikely that there is $M_{24}$ Moonshine in the degeneracies of perturbative heterotic BPS states for the compactification $\mathbb{M}^{1,5} \times T^{4}$.

Clearly, this argument is not completely rigorous mathematically. One might, for example, doubt the accuracy of our numerical expansion, or whether the series is really rapidly convergent, so that there is in fact a solution of the SumDimension game so that the weight in fact converges to $w=-8.5$. This (remote) possibility could in principle be ruled out rigorously as follows: In this case the multiplier system can be deduced using the method of Appendix $\mathbb{F}$. One can then find generators of the relevant space of modular forms and check if it is possible to have an expansion in integers with the first four coefficients given by $Z_{2 A}$. We have not performed this exercise.

\section{F. Deducing The Multiplier System From The Weight}

\section{F.1 Multiplier Systems At Half-Integral Weight}

Suppose that $f(\tau)$ is a modular form of half-integral weight $w$ transforming with a multiplier system under some congruence subgroup $\Gamma$ of $P S L(2, \mathbb{Z})$. Up to a phase $f(\gamma \cdot \tau)$ is $(c \tau+$ $d)^{w} f(\tau)$. Here we state our rules for defining the phase precisely.

Since $\eta(\tau)$ is nonvanishing on the upper half-plane, $g(\tau):=f(\tau) /(\eta(\tau))^{2 w}$ is perfectly well-defined and holomorphic, and $g(\gamma \cdot \tau) / g(\tau)$ for $\gamma \in \Gamma$ has modulus one. On the other hand, it is meromorphic in $\tau$. Therefore it must be a constant in $\tau$. So we write

$$
\frac{g(\gamma \cdot \tau)}{g(\tau)}=\Phi(\gamma)
$$

\footnotetext{
${ }^{10} \mathrm{It}$ is natural to ask if this trick can be used to evaluate modularity of candidate series for $\Gamma_{0}(N)$ for $N>2$ where the weight and multiplier system is unknown. In this case the analogous point would be $\tau_{0}=(1+\mathrm{i}) / N$ and $S T^{N} S \tau_{0}=\tau_{0}-2 / N$, so unless the candidate function satisfies the rather unnatural condition of being a series in powers of $q^{N / 2}$ there is in fact no generalization.
} 
Now it is clear that since the LHS does not depend on $\tau$ we have

$$
\Phi\left(\gamma_{1} \gamma_{2}\right)=\Phi\left(\gamma_{1}\right) \Phi\left(\gamma_{2}\right)
$$

so $\Phi$ must be a unitary character on $\Gamma$.

Now, define:

$$
u(\gamma, \tau):=\eta(\gamma \cdot \tau) / \eta(\tau)
$$

For $\gamma=T^{\ell}$ we have $u\left(T^{\ell}, \tau\right)=e^{2 \pi i \ell / 24}$. In general if

$$
\gamma=\left(\begin{array}{ll}
a & b \\
c & d
\end{array}\right)
$$

with $c \neq 0$ we can write:

$$
u(\gamma, \tau)=\phi(\gamma)(-i(|c| \tau+\operatorname{sign}(c) d))^{1 / 2}
$$

where $\phi(\gamma)$ is a $24^{\text {th }}$ root of 1 and we choose the principal branch of the logarithm. Note that $u(\gamma, \tau)$ is a cocycle: $u\left(\gamma_{1} \gamma_{2}, \tau\right)=u\left(\gamma_{1}, \gamma_{2} \tau\right) u\left(\gamma_{2}, \tau\right)$ (indeed it is also a coboundary). There are explicit formulae for $\phi(\gamma)$ in textbooks on analytic number theory.

We interpret the transformation law for $f(\tau)$ with half-integral weight to be:

$$
f(\gamma \cdot \tau)=(u(\gamma, \tau))^{2 w} \Phi(\gamma) f(\tau)
$$

\section{F.2 Deriving The Multiplier System From the Weight For $\Gamma_{0}(2)$}

Suppose we have a function $f(\tau)$ which is invariant under $\bar{T}: \tau \rightarrow \tau+1$ and transforms with some half-integral weight $w$ under $\bar{S} \bar{T}^{2} \bar{S}$ and hence has weight $w$ with multiplier system under $\bar{\Gamma}_{0}(2)$. We wish to show how to derive the multiplier system given the weight.

We have $u(\bar{T}, \tau)=e^{2 \pi \mathrm{i} / 24}$, and hence

$$
\Phi(\bar{T})=e^{-4 \pi w \mathrm{i} / 24}
$$

Next,

$$
\eta\left(\bar{S} \bar{T}^{2} \bar{S} \cdot \tau\right)=(-\mathrm{i}(2 \tau-1))^{1 / 2} e^{-2 \pi \mathrm{i} / 24} \eta(\tau)
$$

Therefore the transformation law for $f$ under the generator $\bar{S} \bar{T}^{2} \bar{S}$ of $\bar{\Gamma}_{0}(2)$ is

$$
f\left(\bar{S} \bar{T}^{2} \bar{S} \cdot \tau\right)=\left[(-\mathrm{i}(2 \tau-1))^{1 / 2} e^{-2 \pi \mathrm{i} / 24}\right]^{2 w} \Phi\left(\bar{S} \bar{T}^{2} \bar{S}\right) f(\tau)
$$

Now note that if

$$
\tau^{\prime}=\bar{S} \bar{T}^{2} \bar{S} \cdot \tau=\frac{\tau}{1-2 \tau}
$$

then for $\tau_{0}:=(1+\mathrm{i}) / 2$ we have

$$
\tau_{0}^{\prime}=\tau_{0}-1
$$

Therefore, provided $f\left(\tau_{0}\right)$ does not vanish, we get the character on the other generator:

$$
\begin{aligned}
\Phi\left(\bar{S} \bar{T}^{2} \bar{S}\right) & =e^{4 \pi w \mathrm{i} / 24} \\
\Phi(\bar{T}) & =e^{-4 \pi w \mathrm{i} / 24}
\end{aligned}
$$




\section{References}

[1] C. Angelantonj, I. Florakis and B. Pioline, "Rankin-Selberg methods for closed strings on orbifolds," JHEP 1307, 181 (2013) [arXiv:1304.4271 [hep-th]].

[2] P. S. Aspinwall and D. R. Morrison, "String theory on K3 surfaces," In *Greene, B. (ed.), Yau, S.T. (ed.): Mirror symmetry II* 703-716 [hep-th/9404151].

[3] P. S. Aspinwall, "K3 surfaces and string duality," In *Yau, S.T. (ed.): Differential geometry inspired by string theory* 1-95 [hep-th/9611137].

[4] P. S. Aspinwall et al., "Dirichlet branes and mirror symmetry," Clay Mathematics Monographs, 4, Providence, RI: AMS (2009).

[5] T. Banks and N. Seiberg, "Symmetries and Strings in Field Theory and Gravity," Phys. Rev. D 83, 084019 (2011) [arXiv:1011.5120 [hep-th]].

[6] V. Batyrev and M. Kreuzer, "Constructing new Calabi-Yau 3-folds and their mirrors via conifold transitions," Adv. Theor. Math. Phys. 14, no. 3, 879 (2010) doi:10.4310/ATMP.2010.v14.n3.a3 [arXiv:0802.3376 [math.AG]].

[7] R. Borcherds, "Monstrous moonshine and monstrous Lie superalgebras," Invent. Math. 109, No.2 (1992) 405-444.

[8] V. Bouchard and R. Donagi, "On a class of non-simply connected Calabi-Yau threefolds," Commun. Num. Theor. Phys. 2, 1 (2008) [arXiv:0704.3096 [math.AG]].

[9] V. Braun, "Toric Elliptic Fibrations and F-Theory Compactifications," JHEP 1301, 016 (2013) [arXiv:1110.4883 [hep-th]].

[10] T. Bridgeland, "Spaces Of stability conditions," Proc. Sympos. Pure Math., 80, Part 1, Amer. Math., Soc., Providence RI, 2009. [arXiv:math/0611510 [math.AG]].

[11] T. Bridgeland, private communication. See Remark 1.6 of 44 for an quick summary of the argument.

[12] M. C. N. Cheng, J. F. R. Duncan and J. A. Harvey, "Umbral Moonshine," Commun. Num. Theor. Phys. 08, 101 (2014) [arXiv:1204.2779 [math.RT]].

[13] M. C. N. Cheng, J. F. R. Duncan and J. A. Harvey, "Umbral Moonshine and the Niemeier Lattices," Research in the Mathematical Sciences, 2014, vol. 1. arXiv:1307.5793 [math.RT].

[14] M. C. N. Cheng, S. M. Harrison, R. Volpato and M. Zimet, "K3 String Theory, Lattices and Moonshine," arXiv:1612.04404 [hep-th].

[15] W. y. Chuang, D. E. Diaconescu, J. Manschot, G. W. Moore and Y. Soibelman, "Geometric engineering of (framed) BPS states," Adv. Theor. Math. Phys. 18, no. 5, 1063 (2014) [arXiv:1301.3065 [hep-th]].

[16] J. Conway, "A Group Of Order 8,315,553,613,086,720,000," Bull. London Math. Soc., 1 (1969), 79-88.

[17] J. H. Conway and S. P. Norton, "Monstrous Moonshine," Bull. London Math. Soc. 11 (1979) 308-339.

[18] J. H. Conway and S. P. Norton, et. al., Sphere Packings, Lattices and Groups Springer Verlag, Grundlehren der mathematischen Wissenschaften, v. 290. 
[19] A. Dabholkar and J. A. Harvey, "Nonrenormalization of the Superstring Tension," Phys. Rev. Lett. 63, 478 (1989).

[20] A. Dabholkar, F. Denef, G. W. Moore and B. Pioline, "Exact and asymptotic degeneracies of small black holes," JHEP 0508, 021 (2005) [hep-th/0502157].

[21] B. de Wit, M. Rocek and S. Vandoren, "Gauging isometries on hyperKahler cones and quaternion Kahler manifolds," Phys. Lett. B 511, 302 (2001) doi:10.1016/S0370-2693(01)00636-0 [hep-th/0104215].

[22] M. Dine, P. Y. Huet and N. Seiberg, "Large and Small Radius in String Theory," Nucl. Phys. B 322, 301 (1989).

[23] L. J. Dixon, P. H. Ginsparg and J. A. Harvey, "(central charge c) = 1 Superconformal Field Theory," Nucl. Phys. B 306, 470 (1988).

[24] R. Donagi and K. Wendland, "On orbifolds and free fermion constructions," J. Geom. Phys. 59, 942 (2009) [arXiv:0809.0330 [hep-th]].

[25] J. F. R. Duncan, M. J. Griffin and K. Ono, "Proof of the Umbral Moonshine Conjecture," Res. Math. Sci. 2 (2015), Art. 26. [arXiv:1503.01472 [math.RT]].

[26] J. F. R. Duncan, M. H. Mertens and K. Ono, "O’Nan moonshine and arithmetic," arXiv:1702.03516 [math.NT].

[27] T. Eguchi, H. Ooguri and Y. Tachikawa, "Notes on the K3 Surface and the Mathieu group $M_{24}$, Exper. Math. 20, 91 (2011) [arXiv:1004.0956 [hep-th]].

[28] S. Ferrara, J. A. Harvey, A. Strominger and C. Vafa, "Second quantized mirror symmetry," Phys. Lett. B 361, 59 (1995) [hep-th/9505162].

[29] D. Z. Freedman and A. Van Proeyen, Supergravity, Cambridge University Press, 2012.

[30] I. B. Frenkel, J. Lepowsky and A. Meurman, "A natural representation of the Fischer-Griess Monster with the modular function $J$ as character," Proc. Nat. Acad. Sci. U.S.A. 81 (1984) no. 10, Phys. Sci., 3256-3260.

[31] M. R. Gaberdiel, S. Hohenegger and R. Volpato, "Symmetries of K3 sigma models," Commun. Num. Theor. Phys. 6, 1 (2012) [arXiv:1106.4315 [hep-th]].

[32] M. R. Gaberdiel, A. Taormina, R. Volpato and K. Wendland, "A K3 sigma model with $\mathbb{Z}_{2}^{8}$ : $\mathbb{M}_{20}$ symmetry," JHEP 1402, 022 (2014).

[33] T. Gannon, "Much ado about Mathieu," Adv. Math. 301 (2016) 322. [arXiv:1211.5531 [math.RT]].

[34] The GAP Group, GAP - Groups, Algorithms, and Programming, Version 4.8.8; 2017 (https://www.gap-system.org).

[35] P. S. Green, T. Hubsch and C. A. Lutken, "All Hodge Numbers of All Complete Intersection Calabi-Yau Manifolds," Class. Quant. Grav. 6, 105 (1989).

[36] B. R. Greene, D. R. Morrison and A. Strominger, "Black hole condensation and the unification of string vacua," Nucl. Phys. B 451, 109 (1995) [hep-th/9504145].

[37] M. J. Griffin and M. Mertens, "A proof of the Thompson Moonshine Conjecture," arXiv:1607.03078 [math.NT]. 
[38] J. A. Harvey and G. W. Moore, "Algebras, BPS states, and strings," Nucl. Phys. B 463, 315 (1996) [hep-th/9510182].

[39] J. A. Harvey and G. W. Moore, "On the algebras of BPS states," Commun. Math. Phys. 197, 489 (1998) [hep-th/9609017].

[40] J. A. Harvey and B. C. Rayhaun, "Traces of Singular Moduli and Moonshine for the Thompson Group," Commun. Num. Theor. Phys. 10, 23 (2016) [arXiv:1504.08179 [math.RT]].

[41] J. A. Harvey and G. W. Moore, "An Uplifting Discussion of T-Duality," arXiv:1707.08888 [hep-th].

[42] M. Henningson and G. W. Moore, "Threshold corrections in K3 x T2 heterotic string compactifications," Nucl. Phys. B 482, 187 (1996) [hep-th/9608145].

[43] G. Höhn and G. Mason, "The 290 fixed-point sublattices of the Leech lattice," J. Algebra 448 (2016) 618. arXiv:1505.06420v2 [math.GR].

[44] D. Huybrechts, "On derived categories of K3 surfaces, symplectic automorphisms and the Conway group," Adv. Stud. Pure Math., 69, Math. Soc. Japan, 2016. arXiv:1309.6528 [math.AG].

[45] S. Kachru and C. Vafa, "Exact results for $\mathrm{N}=2$ compactifications of heterotic strings," Nucl. Phys. B 450, 69 (1995) [hep-th/9505105].

[46] S. Kachru, N. M. Paquette and R. Volpato, "3D String Theory and Umbral Moonshine," arXiv:1603.07330 [hep-th].

[47] G. Kapustka, "Primitive contractions of Calabi-Yau threefolds. II." J. Lond. Math. Soc. (2) 79 (2009, no. 1, 259.

[48] S. Katz, A. Klemm and R. Pandharipande, "On the motivic stable pairs invariants of K3 surfaces," Progr. Math., 315, K3 surfaces and their moduli, 111-146, Birkhäuser/Springer, 2016. arXiv:1407.3181 [math.AG].

[49] A. Klemm, M. Kreuzer, E. Riegler and E. Scheidegger, "Topological string amplitudes, complete intersection Calabi-Yau spaces and threshold corrections," JHEP 0505, 023 (2005) [hep-th/0410018].

[50] A. Klemm and M. Marino, "Counting BPS states on the enriques Calabi-Yau," Commun. Math. Phys. 280, 27 (2008) [hep-th/0512227].

[51] M. Kontsevich, "Homological Algebra of Mirror Symmetry," Proceedings of the International Congress of Mathematicians, Vol. 1,2 (Zürich, 1994), 120-139, Birkhäuser, Basel, 1995. alg-geom/9411018.

[52] M. Kreuzer and H. Skarke, "On the classification of reflexive polyhedra," Commun. Math. Phys. 185, 495 (1997) [hep-th/9512204].

[53] M. Kreuzer and H. Skarke, "Complete classification of reflexive polyhedra in four-dimensions," Adv. Theor. Math. Phys. 4, 1209 (2002) [hep-th/0002240].

[54] A. K. Lenstra, H. W. Lenstra Jr., L. Lovász, "Factoring polynomials with rational coefficients," Mathematische Annalen, 261, 4 (1982) 515.

[55] J. Lepowsky, "Calculus of twisted vertex operators," Proc. Natl. Acad. Sci. Vol. 82 (1985) pp. 8295-8299. 
[56] R. Miranda and D.R. Morrison, "Embeddings Of Integral Quadratic Forms," http://www.math.ucsb.edu/ drm/manuscripts/eiqf.pdf.

[57] M. Marino and G. W. Moore, "Counting higher genus curves in a Calabi-Yau manifold," Nucl. Phys. B 543, 592 (1999) [hep-th/9808131].

[58] G. W. Moore, "Arithmetic and attractors," hep-th/9807087.

[59] K. S. Narain, M. H. Sarmadi and E. Witten, "A Note on Toroidal Compactification of Heterotic String Theory," Nucl. Phys. B 279, 369 (1987).

[60] K. S. Narain, M. H. Sarmadi and C. Vafa, "Asymmetric Orbifolds," Nucl. Phys. B 288, 551 (1987).

[61] K. S. Narain, M. H. Sarmadi and C. Vafa, "Asymmetric orbifolds: Path integral and operator formulations," Nucl. Phys. B 356, 163 (1991).

[62] V. V. Nikulin, "Integral symmetric bilinear forms and some of their applications," Math. USSR Izvestija, Vol. 14 (1980), No. 1, pp.103-167.

[63] J. Polchinski, "String theory. Vol. 1: An introduction to the bosonic string, Vol.2.: Superstring theory and beyond." Cambridge, UK: Univ. Pr 402 (1998).

[64] W. Taylor, "On the Hodge structure of elliptically fibered Calabi-Yau threefolds," JHEP 1208, 032 (2012) [arXiv:1205.0952 [hep-th]].

[65] W. Taylor and Y. N. Wang, "Non-toric Bases for Elliptic Calabi-Yau Threefolds and 6D F-Theory Vacua," Adv. Theor. Math. Phys. 21 (2017) 1063. arXiv:1504.07689 [hep-th].

[66] J.A. Todd, "A representation of the Mathieu group $M_{24}$ as a collineation group," Annali di Matematica Pura ed Applicata. Serie Quarta 71: 199238.

[67] C. Vafa and E. Witten, "Dual string pairs with $\mathrm{N}=1$ and $\mathrm{N}=2$ supersymmetry in four-dimensions," Nucl. Phys. Proc. Suppl. 46, 225 (1996) [hep-th/9507050].

[68] http://www.math.rwth-aachen.de/ Gabriele.Nebe/LATTICES/. 